\title{
Surface transitions of the semi-infinite Potts model I: the high bulk temperature regime
}

\author{
C. Dobrovolny ${ }^{1}$, L. Laanait ${ }^{2}$, and J. Ruiz ${ }^{3}$
}

\begin{abstract}
We propose a rigorous approach of Semi-Infinite lattice systems illustrated with the study of surface transitions of the semi-infinite Potts model.
\end{abstract}

Key words: Phase transitions, Potts model, Semi-infinite lattice systems.

\footnotetext{
Preprint CPT-2002/P.4465 revised, published in J. Stat. Phys. 114 1269-1302 (2004)

${ }^{1} \mathrm{CPT}$, CNRS, Luminy case 907, F-13288 Marseille Cedex 9, France.

E-mail:dodrovol@cpt.univ-mrs.fr

${ }^{2}$ Ecole Normale supérieure de Rabat, B.P. 5118 Rabat, Morocco

E-mail: laanait@yahoo.fr

${ }^{3}$ CPT, CNRS, Luminy case 907, F-13288 Marseille Cedex 9, France.

E-mail: ruiz@cpt.univ-mrs.fr
} 


\section{Introduction and definitions}

\subsection{Introduction}

Semi-infinite lattice systems with free surface were studied extensively by mean field and numerical approaches. They exhibit a rich variety of critical behaviour depending on the relative strengths of the surface and bulk coupling constants. Ordinary critical behaviour occurs when the surface spins order at the bulk critical temperature as the temperature is lowered. When the surface couplings are sufficiently enhanced, the surface spins order above the bulk critical temperature in a transition known as the surface transition. As the temperature is then lowered through the bulk critical temperature the extraordinary transition takes place. Finally, a special critical behaviour arises if the couplings are adjust such that the bulk and surface transitions coincide. We refer the reader to Binder [3] for a review on the subject. Exact results may be found in [1], 28]. A rigorous approach of the semi-infinite Ising model is proposed in a series of articles by Fröhlich and Pfister, [15][17. In particular, they observed that the surface transition does not occur for temperatures less than the critical bulk temperature (see Figure 1 in [15]).

A new scheme, was found by Lipowsky [26], in the case of the $q$-state Potts model. In the many component limit $q \rightarrow \infty$, both mean field theory and Migdal-Kadanoff renormalization group predict a new phase where the bulk is ordered while the free surface is disordered. The relative strengths of the surface and bulk couplings can be chosen such that when the temperature is lowered, first the bulk spins order through an extraordinary transition and then surface spins order through a surface transition, or a new special transition arises (see diagram Figure 1 in 26]). Intuitively, one can think that the low temperature ordered bulk acts upon the spin on the boundary surface like an external magnetic field. Roughly speaking, one can imagine that this effect modifies the Hamiltonian in the boundary surface by a term containing a magnetic field. Then for the Ising case a surface phase transition cannot occur when the bulk is ordered. However, this observation is not true for the q-state Potts model where it is known for $q$ large that a disordered state arises even in a presence of a magnetic field and coexists with the ordered one aligned with the magnetic field [5].

The aim of this work is to prove that for $q$ large the mean field or renormalization group prediction are indeed correct. We will study both surface phase transitions (of the semi-infinite $q$-state Potts model for bulk dimension 
$d \geq 3$ ), using the Fortuin-Kasteleyn representation of this model. In doing so we think that the method can be extended to a broader class of systems.

Let us sketch out the main scheme of the approach. Since we have to study the surface phase transitions and thus the possible coexistence of "surface phases" (that actually holds), we define following [15] two surface free energies corresponding respectively to the high and low temperature bulk. Here by "surface phases" we mean states invariant under horizontal translations. The ratio of partition functions defining the surface free energies is then expanded using high or low convergent expansion. This leads to horizontally translation invariant systems called hydra models that are then analyzed with the help of Pirogov-Sinai theory [32]. Actually, the theory developed for the study of translation invariant bulk states can be implemented to the case under consideration.

The paper is organized as follow. The surface free energies are defined in the next subsection. In Section 2, we present the Fortuin-Kasteleyn representation and the ground state analysis of the model. Then we give convergent cluster expansions of the bulk partition function in the high and low (bulk) temperature regime. The hydra system for the high temperature bulk case is presented in Subsection 3.1. Subsections 3.2 and 3.3 are devoted to the large $\mathrm{q}$ analysis of the hydra model. Finally, some proofs are postponed to the appendix.

\subsection{Surface free energies}

Consider a ferromagnetic Potts model on the semi-infinite lattice $\mathbb{L}=\mathbb{Z}^{d-1} \times$ $\mathbb{Z}^{+}$of dimension $d \geq 3$. At each site $i=\left\{i_{1}, \ldots, i_{d}\right\} \in \mathbb{L}$, with $i_{\alpha} \in \mathbb{Z}$ for $\alpha=1, \ldots, d-1$ and $i_{d} \in \mathbb{Z}^{+}$, there is a spin variable $\sigma_{i}$ taking its values in the set $\mathcal{Q} \equiv\{0,1, \ldots, q-1\}$. We let $d(i, j)=\max _{\alpha=1, \ldots, d}\left|i_{\alpha}-j_{\alpha}\right|$ be the distance between two sites, $d(i, \Omega)=\min _{j \in \Omega} d(i, j)$ be the distance between the site $i$ and a subset $\Omega \subset \mathbb{L}$, and $d\left(\Omega, \Omega^{\prime}\right)=\min _{i \in \Omega, j \in \Omega^{\prime}} d(i, j)$ be the distance between two subsets of $\mathbb{L}$. The Hamiltonian of the system is given by

$$
H \equiv-\sum_{\langle i, j\rangle} K_{i j} \delta\left(\sigma_{i}, \sigma_{j}\right)
$$

where the sum runs over nearest neighbor pairs $\langle i, j\rangle$ (i.e. at Euclidean distance $\left.d_{\mathrm{E}}(i, j)=1\right)$ of a finite subset $\Omega \subset \mathbb{L}$, and $\delta$ is the Kronecker symbol: $\delta\left(\sigma_{i}, \sigma_{j}\right)=1$ if $\sigma_{i}=\sigma_{j}$, and 0 otherwise. The coupling constants 
$K_{i j}$ can take two values according both $i$ and $j$ belong to the boundary layer $\mathbb{L}_{0} \equiv\left\{i \in \mathbb{L} \mid i_{d}=0\right\}$, or not:

$$
K_{i j}=\left\{\begin{array}{l}
K>0 \text { if }\langle i, j\rangle \subset \mathbb{L}_{0} \\
J>0 \text { otherwise }
\end{array}\right.
$$

The partition function is defined by:

$$
Z^{p}(\Omega) \equiv \sum e^{-\beta H} \chi_{\Omega}^{p}
$$

Here the sum is over configurations $\sigma_{\Omega} \in \mathcal{Q}^{\Omega}, \beta$ is the inverse temperature, and $\chi_{\Omega}^{p}$ is a characteristic function giving the boundary conditions. In particular, we will consider the following boundary conditions:

- the free boundary condition: $\chi_{\Omega}^{\mathrm{f}}=1$

- the ordered boundary condition: $\chi_{\Omega}^{\circ}=\prod_{i \in \partial \Omega} \delta\left(\sigma_{i}, 0\right)$, where the boundary of $\Omega$ is the set of sites of $\Omega$ at distance one to its complement $\partial \Omega=\{i \in \Omega: d(i, \mathbb{L} \backslash \Omega)=1\}$.

- the free boundary condition in the bulk and ordered boundary condition on the surface: $\chi_{\Omega}^{\mathrm{fo}}=\prod_{i \in \partial_{s} \Omega} \delta\left(\sigma_{i}, 0\right)$, where $\partial_{s} \Omega \equiv \partial \Omega \cap \mathbb{L}_{0}$

- the ordered boundary condition in the bulk and free boundary condition on the surface: $\chi_{\Omega}^{\text {of }}=\prod_{i \in \partial_{b} \Omega} \delta\left(\sigma_{i}, 0\right)$, where $\partial_{b} \Omega=\partial \Omega \backslash \partial_{s} \Omega$.

Let us now consider the finite box

$$
\Omega=\left\{i \in \mathbb{L}\left|\max _{\alpha=1, \ldots, d-1}\right| i_{\alpha} \mid \leq L, 0 \leq i_{d} \leq M\right\}
$$

its projection $\Sigma=\Omega \cap \mathbb{L}_{0}=\left\{i \in \Omega \mid i_{d}=0\right\}$ on the boundary layer and its bulk part $\Lambda=\Omega \backslash \Sigma=\left\{i \in \Omega \mid 1 \leq i_{d} \leq M\right\}$.

Following Fröhlich and Pfister [15] we introduce two surface free energies, the free (or disordered) surface free energy with free boundary condition in the bulk,

$$
g_{\mathrm{f}}=-\lim _{L \rightarrow \infty} \frac{1}{|\Sigma|} \lim _{M \rightarrow \infty} \ln \frac{Z^{\mathrm{f}}(\Omega)}{Q^{\mathrm{f}}(\Lambda)}
$$

and the ordered surface free energy corresponding to ordered boundary condition in the bulk,

$$
g_{\mathrm{o}}=-\lim _{L \rightarrow \infty} \frac{1}{|\Sigma|} \lim _{M \rightarrow \infty} \ln \frac{Z^{\circ}(\Omega)}{Q^{\circ}(\Lambda)}
$$


Here $|\Sigma|=(2 L+1)^{d-1}$ is the number of lattice site in $\Sigma$, and $Q^{\mathrm{f}}(\Lambda)$ and $Q^{\circ}(\Lambda)$ are the following bulk partition functions:

$$
\begin{aligned}
Q^{\mathrm{f}}(\Lambda) & =\sum \exp \left\{\beta J \sum_{\langle i, j\rangle \subset \Lambda} \delta\left(\sigma_{i}, \sigma_{j}\right)\right\} \\
Q^{\mathrm{o}}(\Lambda) & =\sum \exp \left\{\beta J \sum_{\langle i, j\rangle \subset \Lambda} \delta\left(\sigma_{i}, \sigma_{j}\right)\right\} \prod_{i \in \partial \Lambda} \delta\left(\sigma_{i}, 0\right)
\end{aligned}
$$

where the two sums are over configurations $\sigma_{\Lambda} \in \mathcal{Q}^{\Lambda}$. The surface free energies do not depend on the boundary condition on the surface, in particular one can replace $Z^{\mathrm{f}}(\Omega)$ by $Z^{\mathrm{fo}}(\Omega)$ in (1.4) and replace $Z^{\mathrm{o}}(\Omega)$ by $Z^{\mathrm{of}}(\Omega)$ in (1.5). The partial derivative of the surface free energy with respect to $\beta K$ represents the mean surface energy. As a result of this paper we get, for $q$ large and $e^{\beta J}-1<q^{1 / d}$, that the mean surface energy $\frac{\partial}{\partial \beta K} g_{\mathrm{f}}$ is discontinuous near $\beta K=\ln \left(1+q^{1 /(d-1)}\right)$.

Namely, let $\langle\cdot\rangle^{p}$ denote the infinite volume expectation corresponding to the boundary condition $p$ :

$$
\langle f\rangle^{p}(\beta J, \beta K)=\lim _{L \rightarrow \infty, M \rightarrow \infty} \frac{1}{Z^{p}(\Omega)} \sum_{\sigma_{\Omega} \in \mathcal{Q}^{\Omega}} f e^{-\beta H} \chi_{\Omega}^{p}
$$

defined for local observable $f$ and let $e^{-\tau}$ be defined by (3.22) below. As a consequence of our main result (Theorem 3.6 in Section 3), we have the following

Corollary 1.1 Assume that $e^{\beta J}-1<q^{1 / d}$ and $q$ is large enough, then there exists a unique value $K_{t}$ such that

(i) for any n.n. pair ij of the surface

$$
\begin{array}{cc}
\left\langle\delta\left(\sigma_{i}, \sigma_{j}\right)\right\rangle^{f}(\beta J, \beta K) \leq O\left(e^{-\tau}\right) & \text { for } \quad K \leq K_{t} \\
\left\langle\delta\left(\sigma_{i}, \sigma_{j}\right)\right\rangle^{f_{o}}(\beta J, \beta K) \geq 1-O\left(e^{-\tau}\right) & \text { for } \quad K \geq K_{t}
\end{array}
$$

(ii) for any n.n. pair ij between the surface and the first layer

$$
\begin{aligned}
\left\langle\delta\left(\sigma_{i}, \sigma_{j}\right)\right\rangle^{f}(\beta J, \beta K) & \leq O\left(e^{-\tau}\right) \\
\left\langle\delta\left(\sigma_{i}, \sigma_{j}\right)\right\rangle^{f o}(\beta J, \beta K) & \leq O\left(e^{-\tau}\right)
\end{aligned}
$$


In that theorem the ratios of the partition functions entering in the definition of the surface free energy $g_{\mathrm{f}}$ (with both $Z^{\mathrm{f}}(\Omega)$ and $Z^{\mathrm{fo}}(\Omega)$ ) are expressed in terms of partition functions of gas of polymers interacting through a twobody hard-core exclusion potential. For $e^{\beta J}-1<q^{1 / d}$ and $q$ large, the associated activities are small according the values of $K$ namely for $K \leq K_{t}$ with the free boundary condition and for $K \geq K_{t}$ with the free-ordered boundary condition. The system is then controlled by convergent cluster expansion.

In the next subsection, we give the expression of the Fortuin-Kasteleyn representation of the partition function $Z^{p}(\Omega)$. We also present, in Theorems 2.1 and 2.2, the high and low temperature expansions of the FortuinKasteleyn representation of the bulk partition function $Q(\Lambda)$ that converge respectively for $e^{\beta J}-1<q^{1 / d}$ and $e^{\beta J}-1>q^{1 / d}$ whenever $q$ is large enough.

\section{Random cluster models}

\subsection{The Fortuin-Kasteleyn (FK) representation}

By using the expansion $e^{\beta K_{i j} \delta\left(\sigma_{i}, \sigma_{j}\right)}=1+\left(e^{\beta K_{i j}}-1\right) \delta\left(\sigma_{i}, \sigma_{j}\right)$, we obtain the Fortuin-Kasteleyn representation [14] of the partition function:

$$
Z^{p}(\Omega)=\sum_{X \subset B(\Omega)} \prod_{\langle i, j\rangle \in X}\left(e^{\beta K_{i j}}-1\right) q^{N_{\Omega}^{p}(X)}
$$

where $B(\Omega)=\{\langle i, j\rangle: i \in \Omega, j \in \Omega\}$ is the set of bonds with both endpoints belonging to $\Omega$, and $N_{\Omega}^{p}(X)$ is the number of connected components (regarding an isolated site $i \in \Omega$ as a component) of a given $X \subset B(\Omega)$. These numbers depend on the considered boundary condition; introducing $S(X)$ as the set of sites that belong to some bond of $X$ and $C(X \mid V)$ as the number of connected components (single sites are not included) of $X$ that do not intersect the set of sites $V$, they are given by:

$$
\begin{aligned}
& N_{\Omega}^{\mathrm{f}}(X)=|\Omega|-|S(X)|+C(X \mid \emptyset) \\
& N_{\Omega}^{\mathrm{o}}(X)=|\Omega|-|S(X) \cup \partial \Omega|+C(X \mid \partial \Omega) \\
& N_{\Omega}^{\mathrm{fo}}(X)=|\Omega|-\left|S(X) \cup \partial_{s} \Omega\right|+C\left(X \mid \partial_{s} \Omega\right) \\
& N_{\Omega}^{\mathrm{of}}(X)=|\Omega|-\left|S(X) \cup \partial_{b} \Omega\right|+C\left(X \mid \partial_{b} \Omega\right)
\end{aligned}
$$


Hereafter $|E|$ denotes the number of elements of the set $E$. The Boltzmann weight in (2.1) can be written in terms of the following Hamiltonian:

$$
H_{\Omega}^{p}(X)=-\beta_{s}\left|X_{s}\right|-\beta_{b}\left|X_{b}\right|-N_{\Omega}^{p}(X)+|\Omega|
$$

where $X_{s}=X \cap B\left(\mathbb{L}_{0}\right), X_{b}=X \backslash X_{s}$, and

$$
\left\{\begin{array}{l}
\beta_{s} \equiv \frac{\ln \left(e^{\beta K}-1\right)}{\ln q} \\
\beta_{b} \equiv \frac{\ln \left(e^{\beta J}-1\right)}{\ln q}
\end{array}\right.
$$

Actually, one has:

$$
Z^{p}(\Omega)=q^{|\Omega|} \sum_{X \subset B(\Omega)} q^{-H_{\Omega}^{p}(X)}
$$

Hence from this representation where the configurations are given by subsets of bonds of the lattice, we are left to analyze a model with an inverse temperature given by $\ln q$ and Hamiltonian given by (2.2) where $\beta_{s}$ and $\beta_{b}$ are independent (real-valued) coupling constants. The next subsection is devoted to the study of the ground states of this model.

\subsection{Ground states}

To study the diagram of ground states of the Fortuin-Kasteleyn representation, it is convenient to introduce the formal Hamiltonian

$$
H(X) \equiv-\beta_{s}\left|X_{s}\right|-\beta_{b}\left|X_{b}\right|+|S(X)|-C(X)
$$

where $C(X)=C(X \mid \emptyset)$ is the number of connected components of $X$, and the relative Hamiltonian

$$
H(Y \mid X) \equiv H(Y)-H(X)
$$

Note, that this last expression makes sense for any configurations $X$ and $Y$ that coincide almost everywhere (a.e.), i.e. that differ only on a finite set of bonds. A configuration $X$ is called ground state, if for any configuration $Y=X$ a.e., one has

$$
H(Y \mid X) \geq 0
$$

For a fixed bond $b$ of the lattice let $n_{b}$ be the occupation number: $n_{b}=1$ if $b$ belongs to $X$ and 0 otherwise. The structure of ground states invariant under horizontal translations is indicated in Fig. 1 (for positive couplings $\beta_{s}$ and $\beta_{b}$ ). 


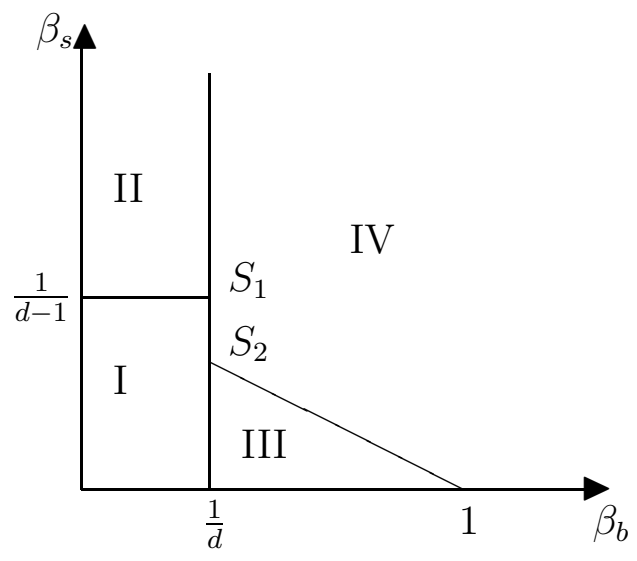

FIGURE 1 : The diagram of ground states.

There are four regions with a unique ground state:

1. In region I $\left(\beta_{b}<\frac{1}{d}, \beta_{s}<\frac{1}{d-1}\right.$, the ground state is the configuration $X^{\mathrm{f}}=\emptyset$.

2. In region II $\left(\beta_{b}<\frac{1}{d}, \beta_{s}>\frac{1}{d-1}\right.$, it is the configuration $X^{\text {fo }}=B\left(\mathbb{L}_{o}\right)$.

3. In region III $\left(\beta_{b}>\frac{1}{d}, \beta_{s}<\frac{1}{d-1}\left(1-\beta_{b}\right)\right.$, it is the configuration $X^{\text {of }}=$ $B\left(\mathbb{L} \backslash \mathbb{L}_{O}\right)$.

4. In region IV $\left(\beta_{b}>\frac{1}{d}, \beta_{s}>\frac{1}{d-1}\left(1-\beta_{b}\right)\right.$, it is the configuration $X^{\mathrm{o}}=$ $B(\mathbb{L})$.

On the separating lines between any two regions the corresponding ground states coexists. The g.s. $X^{\mathrm{f}}$ coexists with $X^{\text {fo }}$ and $X^{\mathrm{o}}$ at the point $S_{1}$ and with $X^{\text {of }}$ and $X^{\mathrm{o}}$ at the point $S_{2}$.

On the part of the line $\beta_{d}=1 / d$ above and on $S_{1}$, there are furthermore infinitely many g.s. that coexist with the previous ones, namely all configurations say $X_{k}, k=2,3, \ldots$, with occupied bonds $n_{b}=1$ below and on the $k$ layer and empty bonds $n_{b}=0$ otherwise. Analogously, on the part of the 
line $\beta_{d}=1 / d$ below and on $S_{2}$ there are also infinitely many g.s. coexisting with the previous ones, namely all configurations $X_{k}^{\prime}, k=2,3, \ldots$, with occupied bonds above and on the $k$ layer and empty bonds otherwise. Finally, when $\beta_{s}=0$ and $\beta_{b} \geq 1$, comes in addition to this list the configuration $\tilde{X}^{\text {of }}=B(\mathbb{L}) \backslash B\left(\mathbb{L}_{o}\right)$.

This suggest that for large $q$ the extraordinary transitions will occur through a sequence of layering transitions. Here we concentrate on the surface transitions, but let us mention a particular study of layering transitions and entropic repulsion for a simplified Solid-On-Solid type model in [9], [7].

\subsection{FK representation in the high temperature regime}

In terms of the Fortuin-Kasteleyn representation, the partition function $Q^{\mathrm{f}}(\Lambda)$ reads:

$$
Q^{\mathrm{f}}(\Lambda)=q^{|\Lambda|} \sum_{Y \subset B(\Lambda)} q^{\beta_{b}|Y|-|S(Y)|+C(Y)}
$$

We will first recall that this system is described by a gas of polymers interacting through two-body hard-core potential and with activity given by (2.8) below.

Indeed, first let us call polymer, a connected subset of $B(\mathbb{L})$ (in the $\mathbb{R}^{d}$ sense). We will use $\mathcal{P}(\Lambda)$ to denote the set of polymers whose bonds belong to $B(\Lambda)$. Two polymers $\gamma_{1}$ and $\gamma_{2}$ are said compatible (we will write $\gamma_{1} \sim \gamma_{2}$ ) if they do not intersect, i.e. if the set $S\left(\gamma_{1}\right) \cap S\left(\gamma_{2}\right)$ is empty. They are said incompatible otherwise and we will use the notation $\gamma_{1} \nsim \gamma_{2}$. A family of polymers is said compatible if any two polymers of the family are compatible and we will use $\mathbf{P}(\Lambda)$ to denote the set of compatible families of polymers $\gamma \in \mathcal{P}(\Lambda)$.

Clearly each subset $Y$ of $B(\Lambda)$ may be decomposed (in a unique way) in a compatible family of polymers $Y=\left\{\gamma_{1}, \ldots, \gamma_{n}\right\}$. Introducing the activity of polymers by

$$
\varphi_{\mathrm{f}}(\gamma)=q^{\beta_{b}|\gamma|-|S(\gamma)|+1}
$$

one has:

$$
Q^{\mathrm{f}}(\Lambda)=q^{|\Lambda|} \sum_{Y \in \mathbf{P}(\Lambda)} \prod_{\gamma \in Y} \varphi_{f}(\gamma)
$$

(with the sum running over compatible families of polymers including the empty-set with weight equal to 1 ). 
We will now introduce multi-indexes in order to write the logarithm of this partition function as a sum over these multi-indexes (see [27]). A multi-index $C$ is a function from the set $\mathcal{P}(\Lambda)$ into the set of non negative integers, and we let $\operatorname{supp} C=\{\gamma \in \mathcal{P}(\Lambda): C(\gamma) \geq 1\}$. We define the truncated functional

$$
\Phi_{0}(C)=\frac{a(C)}{\prod_{\gamma} C(\gamma) !} \prod_{\gamma} \varphi_{f}(\gamma)^{C(\gamma)}
$$

where the factor $a(C)$ is a combinatoric factor defined in terms of the connectivity properties of the graph $G(C)$ with vertices corresponding to $\gamma \in$ $\operatorname{supp} C$ (there are $C(\gamma)$ vertices for each $\gamma \in \operatorname{supp} C$ ) that are connected by an edge whenever the corresponding polymers are incompatible). Namely, $a(C)=0$ and hence $\Phi_{0}(C)=0$ unless $G(C)$ is a connected graph in which case $C$ is called a cluster and

$$
a(C)=\sum_{G \subset G(C)}(-1)^{|e(G)|}
$$

Here the sum goes over connected subgraphs $G$ whose vertices coincide with the vertices of $G(C)$ and $|e(G)|$ is the number of edges of the graph $G$. If the cluster $C$ contains only one polymer, then $a(\gamma)=1$. In other words, the set of all bonds of polymers belonging to a cluster $C$ is connected. The support of a cluster is thus a polymer and it is then convenient to define the following new truncated functional

$$
\Phi(\gamma)=\sum_{C: \operatorname{supp} C=\gamma} \Phi_{0}(C)
$$

As a result we have

Theorem 2.1 Assume that $\beta_{b}<1 / d$ and $c_{0} \nu_{d} q^{-\frac{1}{d}+\beta_{b}} \leq 1$, where $\nu_{d}=(2 d)^{2}$ and $c_{0}=(\sqrt{3}+2) e^{\frac{2}{\sqrt{3}+1}}$, then

$$
Q^{f}(\Lambda)=q^{|\Lambda|} \exp \left\{\sum_{\gamma \in \mathcal{P}(\Lambda)} \Phi(\gamma)\right\}
$$

with a sum running over (non-empty) polymers of $\Lambda$, and the truncated functional $\Phi$ satisfies the estimates

$$
|\Phi(\gamma)| \leq|\gamma|\left(c_{0} \nu_{d} q^{-\frac{1}{d}+\beta_{b}}\right)^{|\gamma|}
$$


Proof. We first notice that easy geometrical observations [23, 24] gives the inequality $\frac{|\gamma|}{d}-|S(\gamma)|+1 \leq 0$ which in turn implies:

$$
\varphi_{\mathrm{f}}(\gamma) \leq q^{-\left(\frac{1}{d}-\beta_{b}\right)|\gamma|}
$$

On the other hand the number of polymers $\gamma$ of length $|\gamma|=n$ containing a given site is less than $\left(\nu_{d}\right)^{n}$. This follows from the fact that for any polymer there exists a path going through the center of every bond at most twice (see [30]). Then we have $\sum_{\gamma \ni x} \varphi_{\mathrm{f}}(\gamma)<\infty$ as soon as $\nu_{d} q^{-\frac{1}{d}+\beta_{b}}<1$.

According ref. [27, under the condition

$$
\varphi_{\mathrm{f}}\left(\gamma_{0}\right) \leq\left(e^{\mu\left(\gamma_{0}\right)}-1\right) \exp \left[-\sum_{\gamma \nsim \gamma_{0}} \mu(\gamma)\right]
$$

where $\mu$ is a positive function, one has

$$
\ln \sum_{Y \in \mathbf{P}(\Lambda)} \prod_{\gamma \in Y} \varphi_{\mathrm{f}}(\gamma)=\sum_{C} \Phi_{0}(C)
$$

where the last sum runs over (non empty) clusters of polymers in $\mathcal{P}(\Lambda)$, and the truncated functional $\Phi_{0}$ satisfies the estimate

$$
\sum_{C: C\left(\gamma_{1}\right) \geq 1}\left|\Phi_{0}(C)\right| \leq \mu\left(\gamma_{1}\right)
$$

We choose $\mu(\gamma)=\left(a \nu_{d}\right)^{-|\gamma|}$ to get by taking into account the above remark on the entropy of polymers

$$
\sum_{\gamma \approx \gamma_{0}} \mu(\gamma) \leq\left|S\left(\gamma_{0}\right)\right| \sum_{n=1}^{\infty} a^{-n} \leq\left(\left|\gamma_{0}\right|+1\right) \frac{a^{-1}}{1-a^{-1}} \leq \frac{2}{a-1}\left|\gamma_{0}\right|
$$

for $a>1$. Since $\mu\left(\gamma_{0}\right) \leq e^{\mu\left(\gamma_{0}\right)}-1$, the bound (2.15) gives that the condition (2.16) will be satisfied whenever

$$
\nu_{d} q^{-\frac{1}{d}+\beta_{b}} a e^{\frac{2}{a-1}} \leq 1
$$

The value $a_{0}=\sqrt{3}+2$ that minimizes the function $a e^{\frac{2}{a-1}}$ provides the conditions given in the theorem. By definition (2.12), the relation (2.17) yields 
(2.13) while the relations (2.18) and (2.19) yield:

$$
\begin{aligned}
\left|\Phi\left(\gamma_{0}\right)\right| & \leq e^{-\lambda\left|\gamma_{0}\right|} \sum_{C: \operatorname{supp} C=\gamma_{0}}\left|\Phi_{0}(C)\right| \prod_{\gamma} e^{\lambda C(\gamma)} \\
& \leq e^{-\lambda\left|\gamma_{0}\right|} \sum_{\gamma \nsim \gamma_{0}} \sum_{C: C(\gamma) \geq 1}\left|\Phi_{0}(C)\right| \prod_{\gamma} e^{\lambda C(\gamma)} \leq \frac{2}{a_{0}-1}\left|\gamma_{0}\right| e^{-\lambda\left|\gamma_{0}\right|}
\end{aligned}
$$

provided $\nu_{d} e^{\lambda} q^{-\frac{1}{d}+\beta_{b}} a_{0} e^{\frac{2}{a_{0}-1}} \leq 1$; we choose for $\lambda$ the value that realizes the equality to get (by taking into account that $\frac{2}{a_{0}-1} \leq 1$ ) the estimate (2.14).

Let us mention that a recent study of these expansions for the Potts model on a general graph, i.e. not restricted to nearest neighbor interactions, is given in ref. [33.

\subsection{FK representation in the low temperature regime}

We now derive an expansion of the bulk partition function $Q^{\circ}(\Lambda)$ at "temperature" $\beta_{b}>\frac{1}{d}$. The FK representation of this partition function reads

$$
Q^{\circ}(\Lambda)=\sum_{Y \subset B(\Lambda)} q^{\beta_{b}|Y|+N_{\Lambda}^{\circ}(Y)}=q^{\beta_{b}|B(\Lambda)|} \sum_{Y \subset B(\Lambda)} q^{-\beta_{b}|B(\Lambda) \backslash Y|+N_{\Lambda}^{\circ}(Y)}
$$

where $N_{\Lambda}^{\circ}(Y)=|\Lambda|-|S(X) \cup \partial \Lambda|+C(X \mid \partial \Lambda)$. The expansion is mainly based on a duality property and we first recall geometrical results on Poincaré and Alexander duality (see e.g. [25, [2], [12, [19]).

We first consider the lattice $\mathbb{Z}^{d}$ and the associated cell-complex $\mathbf{L}$ whose objects $s_{p}$ are called $p$-cells $(0 \leq p \leq d)$ : 0 -cells are vertices, 1 -cells are bonds, 2-cells are plaquettes etc...: a $p$-cell may be represented as $\left(x ; \sigma_{1} e_{1}, \ldots, \sigma_{p} e_{p}\right)$ where $x \in \mathbb{Z}^{d},\left(e_{1}, \ldots, e_{d}\right)$ is an orthonormal base of $\mathbb{R}^{d}$ and $\sigma_{\alpha}= \pm 1, \alpha=$ $1, \ldots, d$. Consider also the dual lattice

$$
\left(\mathbb{Z}^{d}\right)^{*}=\left\{x=\left(x_{1}+\frac{1}{2}, \ldots, x_{d}+\frac{1}{2}\right): x_{\alpha} \in \mathbb{Z}, \alpha=1, \ldots, d\right\}
$$

and the associated cell complex $\mathbf{L}^{*}$. There is a one to-one correspondence

$$
s_{p} \leftrightarrow s_{d-p}^{*}
$$

between $p$-cells of the complex $\mathbf{L}$ and the $d-p$-cells of $\mathbf{L}^{*}$. In particular to each bond $s_{1}$ corresponds the hypercube $s_{d-1}^{*}$ that crosses $s_{1}$ in its middle. 
The dual $E^{*}$ of a subset $E \subset \mathbf{L}$ is the subset of elements of $L^{*}$ that are in the one-to-one correspondence (2.23) with the elements of $E$.

We now turn to the Alexander duality in the particular case under consideration in this paper. Let $Y \subset B(\Lambda)$ be a set of bonds. We define the A-dual of $Y$ as

$$
\widehat{Y}=(B(\Lambda) \backslash Y)^{*}
$$

As a property of Alexander duality one has

$$
\begin{aligned}
|\widehat{Y}| & =|B(\Lambda) \backslash Y| \\
N_{\Lambda}^{\mathrm{o}}(Y) & =N_{\mathrm{cl}}(\widehat{Y})
\end{aligned}
$$

where $N_{\mathrm{cl}}(\widehat{Y})$ denotes the number of independent closed $(d-1)$-surfaces of $\widehat{Y}$. We thus get

$$
Q^{\circ}(\Lambda)=q^{\beta_{b}|B(\Lambda)|} \sum_{\widehat{Y} \subset[B(\Lambda)]^{*}} q^{-\beta_{b}|\widehat{Y}|+N_{\mathrm{cl}}(\widehat{Y})}
$$

Now as in the previous subsection, the system can be described by a gas of polymers interacting through hard core exclusion potential. Indeed, we introduce (hat)-polymers as connected subsets (in the $\mathbb{R}^{d}$ sense) of $(d-1)$ cells of $\mathbf{L}^{*}$ and let $\widehat{\mathcal{P}}(\Lambda)$ denote the set of polymers whose $(d-1)$-cells belong to $[B(\Lambda)]^{*}$. Two polymers $\widehat{\gamma}_{1}$ and $\widehat{\gamma}_{2}$ are compatible (we will write $\widehat{\gamma}_{1} \sim \widehat{\gamma}_{2}$ ) if they do not intersect and incompatible otherwise (we will write $\widehat{\gamma}_{1} \nsim \widehat{\gamma}_{2}$ ). A family of polymers is said compatible if any two polymers of the family are compatible and we will use $\widehat{\mathbf{P}}(\Lambda)$ to denote the set of compatible families of polymers $\widehat{\gamma} \in \mathcal{P}(\Lambda)$. Introducing the activity of polymers by

$$
\varphi_{\mathrm{o}}(\widehat{\gamma})=q^{-\beta_{b}|\widehat{\gamma}|+N_{\mathrm{cl}}(\widehat{\gamma})}
$$

one has:

$$
Q^{\mathrm{o}}(\Lambda)=q^{\beta_{b}|B(\Lambda)|} \sum_{\widehat{Y} \in \widehat{\mathbf{P}}(\Lambda)} \prod_{\widehat{\gamma} \in \widehat{Y}} \varphi_{\mathrm{o}}(\widehat{\gamma})
$$

(with the sum running over compatible families of polymers including the empty-set with weight equal to 1$)$. As in the previous subsection, we introduce multi-indexes $\widehat{C}$ as functions from the set $\widehat{\mathcal{P}}(\Lambda)$ into the set of non negative 
integers, and the truncated functional

$$
\begin{aligned}
\widehat{\Phi}_{0}(\widehat{C}) & =\frac{a(\widehat{C})}{\prod_{\widehat{\gamma}} \widehat{C}(\widehat{\gamma}) !} \prod_{\widehat{\gamma}} \varphi_{\mathrm{o}}(\widehat{\gamma})^{C(\widehat{\gamma})} \\
\widehat{\Phi}(\widehat{\gamma}) & =\sum_{\widehat{C}: \operatorname{supp} \widehat{C}=\widehat{\gamma}} \widehat{\Phi}_{0}(\widehat{C})
\end{aligned}
$$

Here supp $\widehat{C}=\{\widehat{\gamma} \in \widehat{\mathcal{P}}(\Lambda): C(\widehat{\gamma}) \geq 1\}$ and $a(\widehat{C})$ is defined by (2.11) with a graph $G(\widehat{C})$ with vertices corresponding to $\widehat{\gamma} \in \operatorname{supp} \widehat{C}$ that are connected by an edge when the corresponding polymers are incompatible. Again, $\widehat{\Phi}_{0}(\widehat{C})=$ 0 if $\widehat{C}$ is not a cluster i.e. if the set of all $d-1$-cells of polymers belonging to $\operatorname{supp} \widehat{C}$ is not connected.

Theorem 2.2 Assume that $\beta_{b}>1 / d$ and $\widehat{c}_{0} \widehat{\nu}_{d} q^{-\beta_{b}+\frac{1}{d}} \leq 1$, where $\widehat{\nu}_{d}=$ $d^{2} 2^{4(d-1)}$, and $\widehat{c}_{0}=\left[1+2^{d-2}\left(1+\sqrt{1+2^{3-d}}\right)\right] \exp \left[\frac{2}{1+\sqrt{1+2^{3-d}}}\right]$, then

$$
Q^{o}(\Lambda)=e^{\beta_{b}|B(\Lambda)|} \exp \left\{\sum_{\widehat{\gamma} \in \widehat{\mathcal{P}}(\Lambda)} \widehat{\Phi}(\widehat{\gamma})\right\}
$$

with a sum running over (non-empty) polymers, and the truncated functional $\widehat{\Phi}$ satisfies the estimates

$$
|\widehat{\Phi}(\widehat{\gamma})| \leq|\widehat{\gamma}|\left(\widehat{c}_{0} \widehat{\nu}_{d} q^{-\beta_{b}+\frac{1}{d}}\right)^{|\widehat{\gamma}|}
$$

Proof. We first notice that the obvious geometrical inequality $N_{\mathrm{cl}}(\widehat{\gamma}) \leq$ $|\widehat{\gamma}| / d$ implies:

$$
\varphi_{\mathrm{o}}(\widehat{\gamma}) \leq q^{-\left(\beta_{b}-\frac{1}{d}\right)|\widehat{\gamma}|}
$$

On the other hand the number of polymers $\widehat{\gamma}$ of length $|\widehat{\gamma}|=n$ containing a given vertex is less than $\left(\widehat{\nu}_{d}\right)^{n}$. To see it, we first observe that the number of $p$-cells that share a same vertex equals $2^{p}\left(\begin{array}{l}d \\ p\end{array}\right): 2^{p}$ is the choice for the signs of the $\sigma$ 's and the binomial coefficient $\left(\begin{array}{l}d \\ p\end{array}\right)$ is the choice for $p$ vectors $e_{\alpha}$ among $\left(e_{1}, \ldots, e_{d}\right)$. This implies (by duality) that a $(d-1)$-cell contains $2^{d-1}$ vertices and hence that the number of $(d-1)$-cells connected (i.e. sharing at least a vertex) with a given $(d-1)$-cell is less than $2^{(d-1)}\left(d 2^{(d-1)}-1\right)$. Finally, one 
uses the fact that for any polymer of length $n$ there exists a path of length less than $2 n$ going through the center of every $(d-1)$-cell.

For a function $\mu(\widehat{\gamma})=\left(a \widehat{\nu}_{d}\right)^{-|\widehat{\gamma}|}$ we get

$$
\sum_{\widehat{\gamma} \nsim \hat{\gamma}_{0}} \mu(\widehat{\gamma}) \leq \sum_{n=1}^{\infty}\left|S\left(\widehat{\gamma}_{0}\right)\right| a^{-n} \leq \frac{2^{d-1}}{a-1}\left|\widehat{\gamma}_{0}\right|
$$

if $a>1$ and the convergence condition (2.15) for the case under consideration will be satisfied whenever

$$
\widehat{\nu}_{d} q^{-\beta_{b}+\frac{1}{d}} a e^{\frac{2^{d-1}}{a-1}} \leq 1
$$

Here we choose $a_{0}=1+2^{d-2}\left(1+\sqrt{1+2^{3-d}}\right)$ that minimizes the function $a e^{\frac{2^{d-1}}{a-1}}$.

The remainder of the proof is analog to that of Theorem 2.1

\section{Surface transition in the high temperature bulk regime}

\subsection{Hydra model}

To study the surface free energy $g_{\mathrm{f}}$ we shall express the ratio of partition functions entering in its definition (1.4) in terms of a partition function of geometrical objects to be called hydras.

Definition 3.1 A connected set of bonds $\delta \subset B(\Omega)$ is called hydra if there exists a bond of $\delta$ which has one of its endpoint on the boundary surface $\Sigma$ (i.e. if $S(\delta) \cap \Sigma \neq \emptyset$ ).

Any bond of the hydra with one endpoint in the boundary surface $\Sigma$ and one endpoint in the bulk $\Lambda$ is called neck of the hydra.

A connected component of bonds of the hydra with two endpoints in the boundary surface is called foot of the hydra.

A connected component of bonds of the hydra with two endpoints in the bulk is called head of the hydra (heads of hydras are polymers introduced in Subsection 2.3). 


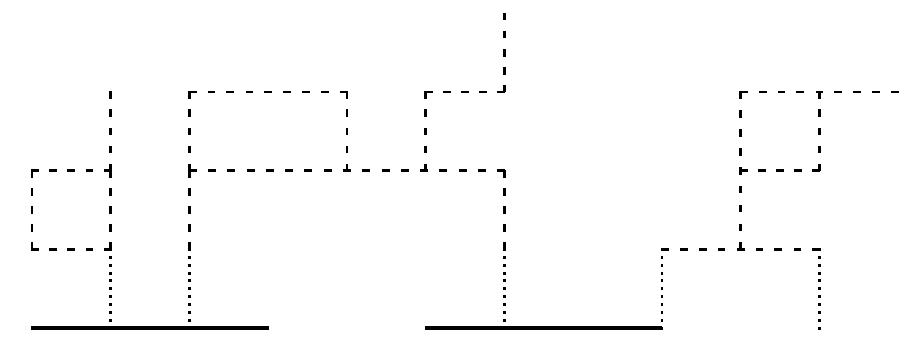

FIGURE 2: A hydra, in two dimensions (a dimension not considered in this paper), with 2 feet (components of full lines), 5 necks (dotted lines), and 3 heads (components of dashed lines).

We let $\mathcal{H}(\Omega)$ denote the set of hydras of $\Omega$. Two hydras $\delta_{1}$ and $\delta_{2}$ are said compatible (we will write $\delta_{1} \sim \delta_{2}$ ) if $S\left(\delta_{1}\right) \cap S\left(\delta_{2}\right) \neq \emptyset$. A family of hydras is said compatible if any two hydras of the family are compatible and we let $\mathbf{H}(\Omega)$ denote the set of compatible families of hydras $\delta \in \mathcal{H}(\Omega)$.

Clearly, a connected subset of bonds included in $B(\Omega)$ is either a hydra $\delta \in \mathcal{H}(\Omega)$ or a polymer $\gamma \in \mathcal{P}(\Lambda)$ (defined in subsection 2.3) and thus any subset of $B \in B(\Omega)$ is a disjoint union of a compatible family of hydras $X \in \mathbf{H}(\Omega)$ with a compatible family of polymers $Y \in \mathbf{P}(\Lambda)$.

Then, the partition function $Z^{p}(\Omega)$ defined by (2.4) reads

$$
Z^{p}(\Omega)=q^{|\Omega|} \sum_{X \in \mathbf{H}(\Omega)} q^{-H_{\Omega}^{p}(X)} \sum_{Y \in \mathbf{P}(\Lambda): Y \sim X} \prod_{\gamma \in Y} \varphi_{\mathrm{f}}(\gamma)
$$

where we take into account that the Boltzmann weight $q^{-H_{\Omega}^{p}(Y)}$ of the subsets $Y \subset B(\Lambda)$ coincides with the bulk one given in (2.7) and the compatibility $Y \sim X$ means $S(Y) \cap S(X)=\emptyset$.

According to Subsection 2.3, the last sum in the RHS of the above formula can be exponentiated as: $\exp \left\{\sum_{\gamma \in \mathcal{P}(\Lambda) ; \gamma \sim X} \Phi(\gamma)\right\}$. Hence dividing the above partition function by the partition function $Q^{\mathrm{f}}(\Lambda)$ we get by taking into 
account Theorem 2.1

$$
\begin{aligned}
\Xi^{p}(\Omega) & \equiv \frac{Z^{p}(\Omega)}{Q^{\mathrm{f}}(\Lambda)} \\
& =q^{|\Sigma|} \sum_{X \in \mathbf{H}(\Omega)} q^{-H_{\Omega}^{p}(X)} \exp \left\{-\sum_{\gamma \in \mathcal{P}(\Lambda) ; \gamma \nsim X} \Phi(\gamma)\right\}
\end{aligned}
$$

Hereafter the incompatibility $\gamma \nsim X$ means $S(\gamma) \cap S(X) \neq \emptyset$.

$\Xi^{p}(\Omega)$ is thus the partition function of a gas of hydras $X=\left\{\delta_{1}, \ldots, \delta_{n}\right\}$ whose activities are defined from the Hamiltonian $H_{\Omega}^{p}(X)$; they interact through hard-core exclusion potential and through a long range interaction potential (decaying exponentially in the distance under the hypothesis of Theorem 2.1) defined on the polymers of the bulk.

Notice that if we neglect this long range potential, the system of hydras will reduce itself to a $(d-1)$ Potts model (in the FK representation), when we moreover restrict to consider only hydras without neck and without head. As well known, the $(d-1)$ Potts model undergoes a temperature driven first order phase transition (at some inverse temperature $\approx(d-1)^{-1} \ln q$ ), whenever $\mathrm{q}$ is large enough and $d \geq 3$. We will show that it is also the case for the hydra model (3.2) implementing the fact that the necks and the heads of hydras modify only weakly their activities and that the long range interaction potential decays exponentially (the needed assumptions are close to those of Theorem 2.1).

To this end it is convenient to first rewrite this potential in terms of a model of aggregates, a technique already developed in many analog situations (see e.g. [18, 11]). Let us introduce the (real-valued) functional

$$
\Psi(\gamma)=e^{-\Phi(\gamma)}-1
$$

defined on polymers $\gamma \in \mathcal{P}(\Lambda)$. An aggregate $A$ is a family of polymers whose support, supp $A=\cup_{\gamma \in A} \gamma$, is connected. Two aggregates $A_{1}$ and $A_{2}$ are said compatible if $\operatorname{supp} A_{1} \cap \operatorname{supp} A_{2}=\emptyset$. A family of aggregates is said compatible if any two aggregates of the family are compatible and we will use $\mathbf{A}(\Lambda)$ to denote the set of compatible families of aggregates. Introducing the statistical weight of aggregates by

$$
\omega(A)=\prod_{\gamma \in A} \Psi(\gamma)
$$


we then get:

$$
\begin{aligned}
\exp \left\{-\sum_{\substack{\gamma \in \mathcal{P}(\Lambda) ; \\
\gamma \nsim X}} \Phi(\gamma)\right\} & =\prod_{\substack{\gamma \in \mathcal{P}(\Lambda) ; \\
\gamma \nsim X}}(1+\Psi(\gamma)) \\
& =\sum_{\mathcal{A} \in \mathbf{A}(\Lambda)} \prod_{\substack{A \in \mathcal{A} \\
A \nsim X}} \omega(A)
\end{aligned}
$$

where $A \nsim X$ means that every polymer of the aggregate $A$ is incompatible with $X$. Since the support of aggregates is a connected set of bonds i.e. a polymer, it is convenient (as it was done for clusters in Subsection 2.3) to sum the statistical weights (3.4) over aggregates with same support. We thus define the weight

$$
\psi(\gamma) \equiv \sum_{A: \operatorname{supp} A=\gamma} \omega(A)
$$

with $A \nsim X$, to get

$$
\Xi^{p}(\Omega)=q^{|\Sigma|} \sum_{X \in \mathbf{H}(\Omega)} q^{-H_{\Omega}^{p}(X)} \sum_{Y \in \mathbf{P}(\Lambda)} \prod_{\substack{\gamma \in Y \\ \gamma \nsim X}} \psi(\gamma)
$$

The system is thus described by two families: a compatible family of hydras and a compatible family of polymers each of these polymers being incompatible with the family of hydras.

The next subsections are devoted to the large $q$ analysis of this model in the frame of Pirogov-Sinai theory. As well known in this theory, it is useful to introduce diluted and crystal partition functions for which an important set of recurrence relation holds (see Lemma 3.2 below). Let us introduce the diluted partition functions for our system .

We define the envelope $E(M)$ of a set of sites of the lattice $\mathbb{L}$ as the set of bonds of $B(\mathbb{L})$ that contain an endpoint in $M$. Next it is convenient to define local observables with which the main terms of the Hamiltonian (the number of bonds and the number of sites) can be written.

Let the energy per site of a configuration $X \subset B(\mathbb{L})$ be:

$$
e_{i}(X)=-\frac{\beta_{s}}{2}\left|E(i) \cap X_{s}\right|-\frac{\beta_{b}}{2}\left|E(i) \cap X_{b}\right|+|i \cap S(X)|
$$


where $X_{s}=X \cap B\left(\mathbb{L}_{0}\right)$ and $X_{b}=X \backslash X_{s}$. We define, for (any) volume $\Omega \subset \mathbb{L}$, the diluted Hamiltonian of a configuration $X=X^{p}$ a.e., as:

$$
H_{\Omega}^{\mathrm{dil}}(X)=\sum_{i \in \Omega} e_{i}(X)-C(X)+C\left(X^{p}\right)
$$

where the number of connected components $C\left(X^{\mathrm{f}}\right)$ of the ground state $X^{\mathrm{f}}$ equals 0 , and the number of connected component $C\left(X^{\mathrm{fo}}\right)$ of the ground state $X^{\text {fo }}$ equals 1 .

Note that even though our model is defined on a $d$-dimensional box $\Omega$ it has a $(d-1)$-dimensional structure and the highest order of the logarithm of partition functions behaves like $O(|\Sigma|)$. It will be convenient to consider $\Omega$ as a set of lines. We let a line $L(x)$ be a cylinder set of sites of $\mathbb{L}$ whose projection on the boundary layer is the site $x$ and whose height is less than a given number $M: L(x)=\left\{i \in \mathbb{L} \mid\left(i_{1}, \ldots, i_{d-1}\right)=x \in \mathbb{L}_{0}, i_{d} \leq M\right\}$. We let $\mathbb{L}_{M}$ be the set of all such lines. For $\Omega \subset \mathbb{L}_{M}$, we let $\Sigma=\Omega \cap \mathbb{L}_{0}$, be its projection on the boundary layer, $\Lambda=\Omega \backslash \Sigma$ and $\|\Omega\|=|\Sigma|$ be the number of lines of $\Omega$. We will use $\mathbf{H}^{p}(\Omega)$ to denote the set of compatible families of hydras defined on the envelope $E(\Omega)$ that coincide with the ground state $X^{p}$ on the envelope $E(\partial \Omega)$ and on the envelope $E\left(\mathbb{L}_{M} \backslash \Omega\right)$, and use $\mathbf{P}^{\text {dil }}(\Lambda)$ to denote the compatible families of polymers defined on $E(\Omega) \backslash(E(\Sigma) \cup E(\partial \Omega))$.

The diluted partition function is defined by

$$
\Xi_{p}^{\text {dil }}(\Omega)=\sum_{X \in \mathbf{H}^{p}(\Omega)} q^{-H_{\Omega}^{\mathrm{dil}}(X)} \sum_{Y \in \mathbf{P}^{\mathrm{dil}}(\Lambda)} \prod_{\substack{\gamma \in Y \\ \gamma \nsim X}} \psi(\gamma)
$$

Notice that the diluted Hamiltonian on ground states reads on set of lines $\Omega \subset \mathbb{L}_{M}$ :

$$
H_{\Omega}^{\text {dil }}\left(X^{p}\right)=e_{p}\|\Omega\|
$$

where

$$
\begin{aligned}
& e_{\mathrm{f}}=0 \\
& e_{\mathrm{fo}}=1-(d-1) \beta_{s}
\end{aligned}
$$

Up to a boundary term $O(\partial \Sigma)$ one has $\ln \Xi^{p}(\Omega)=\|\Omega\| \ln q+\ln \Xi_{p}^{\text {dil }}(\Omega)$ so that

$$
-\lim _{\Omega \uparrow \mathbb{L}} \frac{1}{\|\Omega\|} \ln \Xi_{p}^{\mathrm{dil}}(\Omega)=g_{\mathrm{f}}+\ln q
$$

where $\Omega \uparrow \mathbb{L}$ means that we take first the limit $M \rightarrow \infty$ and then the limit $\Sigma \uparrow \mathbb{L}_{0}$ in the van-Hove or Fisher sense. 
Notice also the following bound

$$
0 \leq \frac{\partial}{\partial \beta_{s}} \ln \Xi_{p}^{\text {dil }}(\Omega) \leq\|\Omega\|(d-1) \ln q
$$

\section{2 contours}

Let $\Omega \subset \mathbb{L}_{M}$ and $(X, Y)$ be a configuration of our system in $\Omega$ : $X \in$ $\mathbf{H}^{p}(\Omega), Y \in \mathbf{P}^{\text {dil }}(\Omega), Y \nsim X$. A site $i \in \Omega$ is called $p$-correct, if $X$ coincides with the ground state $X^{p}$ on the bonds of the envelope $E(i)$ and $Y$ does not contain any bond of $E(i)$. A line is called p-correct if all the sites of the line are p-correct. In other words a line $L$ is f-correct if $X \cap E(L)=\emptyset$ and $Y \cap E(L)=\emptyset$; a line $L$ is fo-correct if $X \cap E(L)=B\left(\mathbb{L}_{0}\right) \cap E(L)$ and $Y \cap E(L)=\emptyset$. Sites and lines that are not p-correct are called incorrect.

The set of incorrect lines of a configuration $(X, Y)$ is called boundary of the configuration $(X, Y)$.

A triplet $\Gamma=\{\operatorname{supp} \Gamma, X(\Gamma), Y(\Gamma)\}$, where supp $\Gamma$ is a maximal connected subset of the boundary of the configuration $(X, Y)$ called support of $\Gamma, X(\Gamma)$ the restriction of $X$ to the envelope $E(\operatorname{supp} \Gamma)$ of the support of $\Gamma$, and $Y(\Gamma)$ the restriction of $Y$ to $E(\operatorname{supp} \Gamma)$, is called contour of the configuration $(X, Y)$. Hereafter a set of sites is called connected if the graph that joins all the sites $i, j$ of this set with $d(i, j) \leq 1$ is connected.

A triplet $\Gamma=\{\operatorname{supp} \Gamma, X(\Gamma), Y(\Gamma)\}$, where $\operatorname{supp} \Gamma$ is a connected set of lines is called contour if there exists a configuration $(X, Y)$ such that $\Gamma$ is a contour of $(X, Y)$. We will use $|\Gamma|$ to denote the number of incorrect points of supp $\Gamma$ and $\|\Gamma\|$ to denote the number of lines of supp $\Gamma$.

Consider the configuration having $\Gamma$ as unique contour; it will be denoted $\left(X^{\Gamma}, Y^{\Gamma}\right)$. Let $L_{p}(\Gamma)$ be the set of p-correct lines of $\mathbb{L}_{M} \backslash$ supp $\Gamma$. Obviously, either a component of $L_{\mathrm{f}}(\Gamma)$ is infinite or a component of $L_{\mathrm{fo}}(\Gamma)$ is infinite. In the first case $\Gamma$ is called contour of the free class or f-contour and in the second case it is called fo-contour. When $\Gamma$ is a p-contour (we will let $\Gamma^{p}$ denote such contours) we use Ext $\Gamma$ to denote the unique infinite component of $L_{p}(\Gamma)$; this component is called exterior of the contour. The set of remaining components of $L_{p}(\Gamma)$ is denoted $\operatorname{Int}_{p} \Gamma$ and the set $L_{m \neq p}(\Gamma)$ is denoted $\operatorname{Int}_{m} \Gamma$. The union $\operatorname{Int} \Gamma=\operatorname{Int}_{\mathrm{f}} \Gamma \cup \operatorname{Int}_{\mathrm{fo}} \Gamma$ is called interior of the contour and $V(\Gamma)=\operatorname{supp} \Gamma \cup \operatorname{Int} \Gamma$.

Two contours $\Gamma_{1}$ and $\Gamma_{2}$ are said compatible if the union of their supports is not connected. They are mutually compatible external contours if $V\left(\Gamma_{1}\right) \subset$ Ext $\Gamma_{2}$ and $V\left(\Gamma_{2}\right) \subset \operatorname{Ext} \Gamma_{1}$. 
We will use $G\left(\Gamma^{p}\right)$ to denote the set of configurations having $\Gamma^{p}$ as unique external contour. The crystal partition function is then defined by :

$$
\Xi^{\mathrm{cr}}\left(\Gamma^{p}\right)=\sum_{(X, Y) \in G\left(\Gamma^{p}\right)} q^{-H_{V\left(\Gamma^{p}\right)}^{\mathrm{dil}}(X)} \prod_{\gamma \in Y} \psi(\gamma)
$$

Lemma 3.2 The following set of recurrence equations holds :

$$
\Xi_{p}^{d i l}(\Omega)=\sum_{\left\{\Gamma_{1}^{p}, \ldots, \Gamma_{n}^{p}\right\}_{\text {ext }}} q^{-e_{p}\|E x t\|} \prod_{i=1}^{n} \Xi^{c r}\left(\Gamma_{i}^{p}\right)
$$

Here the sum is over families $\left\{\Gamma_{1}^{p}, \ldots, \Gamma_{n}^{p}\right\}_{\text {ext }}$ of mutually compatible external contours in $\Omega\left(\operatorname{supp} \Gamma_{i}^{p} \subset \Omega_{\text {int }}=\left\{i \in \Omega: d\left(i, \mathbb{L}_{M} \backslash \Omega\right)>1\right\}\right), \|$ Ext $\|=\| \Omega \|-$ $\sum_{i}\left\|V\left(\Gamma_{i}^{p}\right)\right\|$ where $\left\|V\left(\Gamma_{i}^{p}\right)\right\|$ is the number of lines of $V\left(\Gamma_{i}^{p}\right)$;

$$
\Xi^{c r}\left(\Gamma^{p}\right)=\varrho\left(\Gamma^{p}\right) \prod_{m \in\{f, f o\}} \Xi_{m}^{d i l}\left(\operatorname{Int}_{m} \Gamma^{p}\right)
$$

where:

$$
\varrho\left(\Gamma^{p}\right) \equiv q^{-H_{s u p p}^{d i l} \Gamma^{p}\left(X^{\Gamma^{p}}\right)} \prod_{\gamma \in Y_{\Gamma^{p}}} \psi(\gamma)
$$

Proof. We have only to observe that for any $X \in \mathbf{H}^{p}(\Omega)$

$$
H_{\Omega}^{\mathrm{dil}}(X)=\sum_{\Gamma} H_{\mathrm{supp} \Gamma}^{\mathrm{dil}}\left(X^{\Gamma}\right)+\sum_{p} e_{p}\left\|L_{p}(X) \cap \Omega\right\|
$$

where the sum is over all contours of the boundary of the configuration $(X, Y=\emptyset)$ and $\left\|L_{p}(X) \cap \Omega\right\|$ is the number of $p$-correct lines inside $\Omega$ of this configuration.

Lemma 3.2 gives the following expansion for the partition function

$$
\Xi_{p}^{\text {dil }}(\Omega)=q^{-e_{p}\|\Omega\|} \sum_{\left\{\Gamma_{1}^{p}, \ldots, \Gamma_{n}^{p}\right\}_{\text {comp }}} \prod_{i=1}^{n} z\left(\Gamma_{i}^{p}\right)
$$

where the sum is now over families of compatibles contours of the same class and

$$
z\left(\Gamma^{p}\right)=\varrho\left(\Gamma^{p}\right) q^{e_{p}\left\|\Gamma^{p}\right\|} \frac{\Xi_{m}^{\operatorname{dil}}\left(\operatorname{Int}_{m} \Gamma^{p}\right)}{\Xi_{p}^{\operatorname{dil}}\left(\operatorname{Int}_{m} \Gamma^{p}\right)}
$$


with $m \neq p$ (and $\left\|\Gamma^{p}\right\|$ is the number of lines of $\operatorname{supp} \Gamma^{p}$ ).

To control the behavior of our system, we need to show Peierls condition, that means that $\varrho\left(\Gamma^{p}\right) q^{e_{p}}\left\|\Gamma^{p}\right\|$ has good decaying properties with respect to the number of incorrect points $\left|\Gamma^{p}\right|$ of $\operatorname{supp} \Gamma^{p}$. In fact we shall consider the modified Peierls condition introduced in ref. [21] where $e_{p}$ is replaced by $\underline{e}=\min \left(e_{\mathrm{f}}, e_{\mathrm{fo}}\right)$. Let

$$
e^{-\tau}=\left(2^{2 d-1} q^{-\frac{1}{2(d-1)}}+24 c \nu_{d}^{3} q^{-\left(\frac{1}{d}-\beta_{b}\right)}\right) \frac{1}{1-6 c \nu_{d}^{3} q^{-\left(\frac{1}{d}-\beta_{b}\right)}}
$$

where $c=8 e(e-1)(\sqrt{3}+2) e^{\frac{2}{\sqrt{3}+1}}$ and $\nu_{d}=(2 d)^{2}$. We have the following

Proposition 3.3 (Peierls condition) Let $\Omega \subset \mathbb{L}_{M}$ be a finite connected set of lines, assume that $\beta_{b}<\frac{1}{d}$, and $6 c \nu_{d}^{3} q^{-\frac{1}{d}+\beta_{b}}<1$, then for all $\beta_{s} \in \mathbb{R}$ :

$$
\sum_{\Gamma^{p}: \text { supp } \Gamma^{p}=S}\left|\varrho\left(\Gamma^{p}\right)\right| q^{\underline{e}\left\|\Gamma^{p}\right\|} \leq e^{-\tau\|S\|}
$$

where $\|S\|$ is the number of lines of $S$.

The proof is postponed to the Appendix.

The recurrence equations of Lemma 3.2 play in our case the role of Lemma 2.3 from Ref. [32. Together with the Peierls estimates 3.3, they allow to study the states invariant under horizontal translation (HTIS) of the hydra system by applying Pirogov-Sinai theory. Here we do not need any extension [10] 6] developed for interacting contours. Actually we have non interacting contours and only a standard form of the theory is required. We present it in the next subsection. We chose Zahradnik's approach 34 with the notion of truncated contour models including some improvements from [20]. We are dealing with complex activities, a situation analyzed in Refs. [35], 4] and we mainly follow the presentation given in that last reference.

\subsection{Diagram of horizontal translation invariant states}

To state our result, we first define the functional

$$
K_{p}(S)=\sum_{\Gamma^{p}: \operatorname{supp} \Gamma^{p}=S} z\left(\Gamma^{p}\right)
$$


Consider the partition function $\Xi_{p}^{\text {dil }}(\Omega)$ (3.20) and for a compatible family $\left\{\Gamma_{1}^{p}, \ldots, \Gamma_{n}^{p}\right\}_{\text {comp }}$ of $p$-contours, denote by $S_{1}, \ldots, S_{n}$ their respective supports. By summing over all contours with the same support this partition function can be written as the partition function of a gas of polymers $S$ with activity $K_{p}(S)=\sum_{\Gamma^{p}: \operatorname{supp} \Gamma^{p}=S} z\left(\Gamma^{p}\right)$ interacting through hard-core exclusion potential:

$$
\Xi_{p}^{\mathrm{dil}}(\Omega)=q^{-e_{p}\|\Omega\|} \sum_{\left\{S_{1}, \ldots, S_{n}\right\}_{\mathrm{comp}}} \prod_{i=1}^{n} K_{p}\left(S_{i}\right)
$$

Here $\left\{S_{1}, \ldots, S_{n}\right\}_{\text {comp }}$ denotes compatible families of polymers, that is $d\left(S_{i}, S_{j}\right)>1$ for every two $S_{i}$ and $S_{j}$ in the family: recall that by definitions of contours a polymer $S$ is a set of lines whose graph that joins all the points of the lines of $S$ at distance $d(i, j) \leq 1$ is connected.

Next we define auxiliary models, namely the truncated contour models associated to each boundary conditions.

Definition 3.4 A truncated contour functional is defined as

$$
K_{p}^{\prime}(S)= \begin{cases}K_{p}(S) & \text { if }\left\|K_{p}(S)\right\| \leq e^{-\alpha\|S\|} \\ 0 & \text { otherwise }\end{cases}
$$

where $\left\|K_{p}(S)\right\|=\sum_{\Gamma^{p} \text { :supp } \Gamma^{p}=S}\left|z\left(\Gamma^{p}\right)\right|$, and $\alpha>0$ is some positive parameter to be chosen later (see Theorem $\mathbf{3 . 6}$ below).

Definition 3.5 The collection $\{S, p\}$ of all $p$-contours $\Gamma^{p}$ with support supp $\Gamma^{p}=S$ is called stable if

$$
\left\|K_{p}(S)\right\| \leq e^{-\alpha\|S\|}
$$

i.e. if $K_{p}(S)=K_{p}^{\prime}(S)$.

We define the truncated partition function $\Xi_{p}^{\prime}(\Omega)$ as the partition function obtained from $\Xi_{p}^{\text {dil }}(\Omega)$ by leaving out unstable collections of contours, namely

$$
\begin{aligned}
\Xi_{p}^{\prime}(\Omega) & =q^{-e_{p}\|\Omega\|} \sum_{\left\{\Gamma_{1}^{p}, \ldots, \Gamma_{n}^{p}\right\}_{\mathrm{comp}}}^{\prime} \prod_{i=1}^{n} z\left(\Gamma_{i}^{p}\right) \\
& =q^{-e_{p}\|\Omega\|} \sum_{\left\{S_{1}, \ldots, S_{n}\right\}_{\mathrm{comp}}} \prod_{i=1}^{n} K_{p}^{\prime}\left(S_{i}\right)
\end{aligned}
$$


Here the sum goes over compatible families of stable collections of contours. Let

$$
h_{p}=-\lim _{\Omega \rightarrow L} \frac{1}{\|\Omega\|} \ln \Xi_{p}^{\prime}(\Omega)
$$

be the metastable free energy of the truncated partition function $\Xi_{p}^{\prime}(\Omega)$.

For $\alpha$ large enough, the thermodynamic limit (3.29) can be controlled by a convergent cluster expansion. We conclude the existence of $h_{p}$, together with the bounds

$$
\begin{aligned}
e^{-\kappa e^{-\alpha}\left|\partial_{s} \Omega\right|} & \leq \Xi_{p}^{\prime}(\Omega) e^{h_{p}\|\Omega\|} \leq e^{\kappa e^{-\alpha}\left|\partial_{s} \Omega\right|} \\
\left|h_{p}-e_{p} \ln q\right| & \leq \kappa e^{-\alpha}
\end{aligned}
$$

shown below. Here $\kappa=\kappa_{\mathrm{cl}}\left(\chi^{\prime}\right)^{2}$ where $\kappa_{\mathrm{cl}}=\frac{\sqrt{5}+3}{2} e^{\frac{2}{\sqrt{5}+1}}$ is the cluster constant [20] and $\kappa^{\prime}=3^{d-1}-1 ; \partial_{s} \Omega=\partial \Omega \cap \mathbb{L}_{0}$ in the way defined in Subsection 1.2

Theorem 3.6 Assume $\beta_{b}<1 / d$ and $q$ is large enough so that $e^{-\alpha} \equiv$ $e^{-\tau+2 \kappa^{\prime}+3}<\frac{0.7}{\kappa \kappa^{\prime}}$, then there exists a unique $\beta_{s}^{t}=\frac{1}{d-1}+O\left(e^{-\tau}\right)$ such that :

(i) for $\beta_{s}=\beta_{s}^{t}$

$$
\Xi_{p}^{d i l}(\Omega)=\Xi_{p}^{\prime}(\Omega)
$$

for both boundary conditions $p=f$ and $p=o f$, and the free energy of the hydra model is given by $g_{f}+\ln q=h_{f}=h_{f_{o}}$.

(ii) for $\beta_{s}<\beta_{s}^{t}$

$$
\Xi_{f}^{d i l}(\Omega)=\Xi_{f}^{\prime}(\Omega)
$$

and $g_{f}+\ln q=h_{f}<h_{f_{o}}$

(iii) for $\beta_{s}>\beta_{s}^{t}$

$$
\Xi_{f o}^{d i l}(\Omega)=\Xi_{f o}^{\prime}(\Omega)
$$

and $g_{f}+\ln q=h_{f o}<h_{f}$

Let us introduce the Gibbs states $\langle\cdot\rangle^{p}$, associated to the partition functions $\Xi_{p}^{\text {dil }}$. Theorems above show also that at $\beta_{s}=\beta_{s}^{t}$

$$
\begin{aligned}
& \left\langle n_{b}\right\rangle^{\mathrm{f}}=O\left(e^{-\tau}\right) \\
& \left\langle n_{b}\right\rangle^{\text {fo }}=1-O\left(e^{-\tau}\right)
\end{aligned}
$$


for any bond $b$ of the boundary layer while

$$
\begin{aligned}
& \left\langle n_{b^{\prime}}\right\rangle^{\mathrm{f}}=O\left(e^{-\tau}\right) \\
& \left\langle n_{b^{\prime}}\right\rangle^{\text {fo }}=O\left(e^{-\tau}\right)
\end{aligned}
$$

for any bond $b^{\prime}$ between the boundary layer and the first layer. This is because, with free (f) boundary conditions, bonds $b$ of the boundary layer and bonds $b^{\prime}$ of between the boundary and first layer are occupied only if there is a free contour surrounding them and that the correlation functions are controlled by the contour model cluster expansion. With free-ordered (fo) boundary conditions, bonds $b$ are empty and bonds $b^{\prime}$ are occupied only if there is a fo-contour surrounding it and again the correlations are controlled by cluster expansion.

This shows in particular that the derivative $\frac{\partial}{\partial K} g_{\mathrm{f}}$ of the free energy $g_{\mathrm{f}}$ with respect to the surface coupling constant $K$ is discontinuous near $K=$ $\beta^{-1} \ln \left(1+q^{\frac{1}{d-1}}\right)$.

Obviously, the first relations of (3.32) and (3.33) hold true for any $\beta_{s} \leq \beta_{s}^{t}$ while the second relations of (3.32) and (3.33) hold true for any $\beta_{s} \geq \beta_{s}^{t}$. Notice also that the estimate (3.33) on the mean values of the bonds $b^{\prime}$ between the boundary and first layer can be extended to the bonds of the bulk.

\section{Outline of proof of Theorem 3.6}

The first step is to prove the inequalities (3.30) and (3.31). To exponentiate the partition function

$$
\mathcal{Z}_{p}(\Omega)=q^{e_{p}\|\Omega\|} \Xi_{p}^{\prime}(\Omega)=\sum_{\left\{S_{1}, \ldots, S_{n}\right\}_{\mathrm{comp}}} \prod_{i=1}^{n} K_{p}^{\prime}\left(S_{i}\right)
$$

we define the truncated functional $\Phi^{T}$ associated to $K_{p}^{\prime}$

$$
\Phi^{T}(X)=\frac{a(X)}{\prod_{\gamma} X(S) !} \prod_{S} K_{p}^{\prime}(S)^{X(S)}
$$

defined on the multi-indexes $X$ associated to the polymers: a multi-index is a function from the set of polymers into the set of non negative integers, and $a(X)$ is defined as in (2.11).

The number of polymers $S$ with number of lines $\|S\|=n$ and containing a given line can be bounded by $\nu^{n}$ where $\nu=\left(3^{d-1}-1\right)^{2}$. Actually, There 
exists at most $3^{d-1}-1$ lines at distance 1 of a given line and for any connected set of lines there exists a graph going through every line at most twice.

As a result of the standard cluster expansion [20, 27, we get for $\kappa e^{-\alpha}<1$

$$
\begin{aligned}
\mathcal{Z}_{p}(\Omega) & =\exp \left\{\sum_{X} \Phi_{T}(X)\right\} \\
& =\exp \left\{\|\Omega\| \sum_{X: \operatorname{supp} X \ni L} \frac{\Phi^{T}(X)}{\|\operatorname{supp} X\|}-\sum_{X}^{*} \frac{\|\operatorname{supp} X \cap \Omega\|}{\|\operatorname{supp} X\|} \Phi^{T}(X)\right\}
\end{aligned}
$$

Here, the sum in (3.36) is over multi-indexes whose support supp $X=$ $\{S: X(S) \geq 1\}$ belongs to $\Omega$, the first sum in (3.37) is over all multi-indexes $X$ whose support contains a given line $L$, and the second sum in (3.37) runs over multi-indexes whose support intersects both $\Omega$ and its complement $\mathbb{L}_{M} \backslash \Omega$.

The series $\sum_{X: \text { :supp } X э L}\left|\Phi^{T}(X)\right|$ is absolutely convergent and satisfies the bound

$$
\sum_{X: \operatorname{supp} X \ni L}\left|\Phi^{T}(X)\right| \leq \kappa e^{-\alpha}
$$

that immediately gives (3.30) and (3.31).

The second step closely follows the Borgs Imbrie paper 4].

We put

$$
a_{p}=h_{p}-\min _{m} h_{m}
$$

The boundary condition $p$ is then called stable if $a_{p}=0$. Our task is now to show that if the boundary condition $p$ is stable, then all collections of $p$-contours are stable implying that $\Xi_{p}^{\prime}(\Omega)$ coincides with $\Xi_{p}^{\text {dil }}(\Omega)$.

We notice that when $a_{p} \leq 1$ and the hypotheses of Proposition 3.3 hold, then the condition

$$
\frac{\Xi_{m}^{\text {dil }}\left(\operatorname{Int}_{m} \Gamma^{p}\right)}{\Xi_{p}^{\text {dil }}\left(\operatorname{Int}_{m} \Gamma^{p}\right)} \leq e^{2\left|\partial_{s} \operatorname{Int}_{m} \Gamma^{p}\right|}
$$

for all contours $\Gamma^{p}$ with support $S$ ensures that the collection $\{S, p\}$ is stable, provided

Indeed since

$$
e^{-\alpha}=e^{-\tau+2 \kappa^{\prime}+3}<\frac{1}{\kappa}
$$

$$
z\left(\Gamma^{p}\right)=\varrho\left(\Gamma^{p}\right) q^{\underline{e}\left\|\Gamma^{p}\right\|} q^{\left(e_{p}-\underline{e}\right)\left\|\Gamma^{p}\right\|} \frac{\Xi_{m}^{\operatorname{dil}}\left(\operatorname{Int}_{m} \Gamma^{p}\right)}{\Xi_{p}^{\operatorname{dil}}\left(\operatorname{Int}_{m} \Gamma^{p}\right)}
$$


we get, by Proposition 3.3, using $\left(e_{p}-\underline{e}\right) \ln q \leq a_{p}+2 \kappa e^{-\alpha} \leq 3$ (see (B.31)),

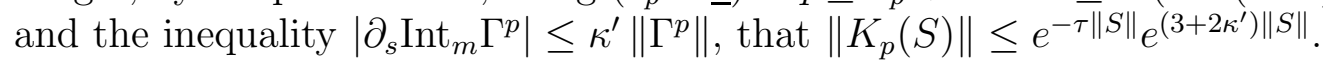

We then show inductively on $\operatorname{diam} \Omega=\max _{i, j \in \Omega \cap \mathbb{L}_{0}} d(i, j)$, that

(i) if $a_{m} \operatorname{diam} \Omega \leq 1$, and $a_{p}=0$, then

$$
\left|\frac{\Xi_{P}^{\operatorname{dil}}(\Omega)}{\Xi_{m}^{\operatorname{dil}}(\Omega)}\right| \leq e^{a_{m}\|\Omega\|+2 \kappa e^{-\alpha}\left|\partial_{s} \Omega\right|}
$$

(ii) if $a_{p}=0$, then

$$
\left|\frac{\Xi_{m}^{\operatorname{dil}}(\Omega)}{\Xi_{p}^{\operatorname{dil}}(\Omega)}\right| \leq e^{3 \kappa e^{-\alpha}\left|\partial_{s} \Omega\right|}
$$

(iii) if $a_{m} \operatorname{diam} \Omega \leq 1$, then

$$
\left|\frac{\Xi_{\tilde{m}}^{\operatorname{dil}}(\Omega)}{\Xi_{m}^{\operatorname{dil}}(\Omega)}\right| \leq e^{\left(1+5 \kappa e^{-\alpha}\right)\left|\partial_{s} \Omega\right|}
$$

The proof is analog to that of Theorem 3.1 in [4] using our previous estimates and thus we omit it. It gives that for $\beta_{b}<1 / d$ and $q$ is large enough so that $e^{-\tau+2 \kappa^{\prime}+3}=e^{-\alpha}<\frac{1}{5 \kappa}$, then if $a_{p}=0$ all collections of $p$ contours are stable.

For our last step, it remains to show that there exists a unique $\beta_{s}^{t}=$ $\frac{1}{d-1}+O\left(e^{-\tau}\right)$ such that:

$$
\begin{array}{ll}
a_{\mathrm{f}}=0 \text { and } a_{\mathrm{fo}}=0 & \text { for } \beta_{s}=\beta_{s}^{t} \\
a_{\mathrm{f}}=0 \text { and } a_{\mathrm{fo}}>0 & \text { for } \beta_{s}<\beta_{s}^{t} \\
a_{\mathrm{f}}>0 \text { and } a_{\mathrm{fo}}=0 & \text { for } \beta_{s}>\beta_{s}^{t}
\end{array}
$$

This is a consequence of the fact that the free energy $\frac{-1}{\|\Omega\| \ln q} \ln \mathcal{Z}_{p}(\Omega)$ is a Lipschitz function of $\beta_{s}$ uniformly in $\Omega$ with a small Lipschitz constant (when $\tau$ is large, one has a good control on the one sided derivative $\frac{\partial}{\partial^{ \pm} \beta_{s}} K_{p}^{\prime}(S)$ of the activities associated to the partition functions $\mathcal{Z}_{p}(\Omega)$ ).

Lemma 3.7 Assume that $\kappa e^{-\alpha}<\frac{c}{\kappa^{\prime}} \leq \frac{0.7}{\kappa^{\prime}}$, then

$$
\left|\frac{1}{\|\Omega\| \ln q} \frac{\partial}{\partial^{ \pm} \beta_{s}} \ln \mathcal{Z}_{p}(\Omega)\right| \leq(d-1) \frac{a_{c} \kappa e^{-\alpha}}{1-a_{c} \kappa e^{-\alpha}}
$$

where $a_{c}=\frac{e}{\kappa_{c l}} e^{9 c / 8}$. 


\section{Proof.}

By virtue of relation (3.21) one gets

$$
\begin{array}{r}
\frac{\partial}{\partial \beta_{s}} K_{p}(S)=\sum_{\Gamma^{p}: \text { supp } \Gamma^{p}=S}\left(\frac{\partial}{\partial \beta_{s}} \ln \varrho\left(\Gamma^{p}\right)\right. \\
+\frac{\partial}{\partial \beta_{s}} \ln q^{e_{p}\left\|\Gamma^{p}\right\|} \\
\left.+\frac{\partial}{\partial \beta_{s}} \ln \frac{\Xi_{m}^{\operatorname{dil}_{m}}\left(\operatorname{Int}_{m} \Gamma^{p}\right)}{\Xi_{p}^{\operatorname{dil}}\left(\operatorname{Int}_{m} \Gamma^{p}\right)}\right) z\left(\Gamma^{p}\right)
\end{array}
$$

Since $\frac{\partial e_{p}}{\partial \beta_{s}}=0$ or $\frac{\partial e_{p}}{\partial \beta_{s}}=-(d-1)$ (see (3.12) $)$, the bound (A.15) on the first term inside the parenthesis gives that $\left|\frac{\partial}{\partial \beta_{s}} \ln \varrho\left(\Gamma^{p}\right)+\frac{\partial}{\partial \beta_{s}} \ln q^{e_{p}}\left\|\Gamma^{p}\right\|\right| \leq$ $\left\|\Gamma^{p}\right\|(d-1) \ln q$. This inequality together with the bound (3.14) on the third term inside the parenthesis leads to

$$
\begin{aligned}
\left|\frac{\partial}{\partial \beta_{s}} K_{p}(S)\right| & \leq \sum_{\Gamma^{p}: \operatorname{supp} \Gamma^{p}=S}\left[\left\|\Gamma^{p}\right\|(d-1) \ln q+\left\|\operatorname{Int}_{m} \Gamma^{p}\right\|(d-1) \ln q\right]\left|z\left(\Gamma^{p}\right)\right| \\
& \leq[\|V(S)\|(d-1) \ln q] e^{-\alpha\|S\|}
\end{aligned}
$$

where $V(S) \equiv V\left(\Gamma^{p}\right)=\operatorname{Int} \Gamma^{p} \cup \Gamma^{p}$.

Taking into account the fact that either the one-sided derivative $\frac{\partial}{\partial^{ \pm} \beta_{s}} K_{p}^{\prime}(S)=$ 0 or $\frac{\partial}{\partial^{ \pm} \beta_{s}} K_{p}^{\prime}(S) \leq \frac{\partial}{\partial \beta_{s}} K_{p}(S)$ we get that the left-hand side of (3.49) is bounded as

$$
\begin{aligned}
& \left|\frac{1}{\|\Omega\|} \frac{\partial}{\partial^{ \pm} \beta_{s}} \ln \mathcal{Z}_{p}(\Omega)\right| \leq \sum_{S: S \ni L}\left|\frac{\partial}{\partial^{ \pm} \beta_{s}} K_{p}^{\prime}(S)\right| \times\left|\frac{\sum_{\left\{S_{1}, \ldots, S_{n}\right\}_{\text {comp }}}^{*} K_{p}^{\prime}(S)}{\mathcal{Z}_{p}(\Omega)}\right| \\
& \leq(d-1) \ln q \sum_{S: S \ni L}\|V(S)\| e^{-\alpha\|S\|} \exp \left\{-\sum_{X: \operatorname{supp} X \cap \bar{S}=\varnothing} \Phi^{T}(X)\right\} \\
& \leq(d-1) \ln q \sum_{S: S \ni L}\|V(S)\| e^{-\alpha\|S\|} e^{\left(1+\kappa^{\prime}\right) \kappa e^{-\alpha}\|S\|}
\end{aligned}
$$

where the sum $\sum^{*}$ is over all families $\left\{S_{1}, \ldots, S_{n}\right\}_{\text {comp }}$ compatible with $S$ and $\bar{S}=S \cup\{L: d(L, S)=1\}$ is the set of lines at distance less or equal to 1 from $S$.

The last inequality leads to the lemma by inserting the bound

$$
\|V(S)\| \leq\|S\| \operatorname{diam} S \leq\|S\|^{2} \leq e^{\|S\|}
$$


and using the fact that the number of polymers with number of lines $\|S\|=n$ and containing a given line can be bounded by $\nu^{n}=\left(\kappa^{\prime}\right)^{2 n}$.

To prove relations (3.46, 3.48), we consider the energy difference $\frac{e_{\mathrm{fo}}-e_{\mathrm{f}}}{d-1}=$ $\beta_{s}-\frac{1}{d-1}$. As a function of $\beta_{s}$ it is obviously increasing, negative for $\beta_{s}<\frac{1}{d-1}$, positive for $\beta_{s}>\frac{1}{d-1}$, moreover it intersects the horizontal coordinate axis only at one point $\beta_{s}=\frac{1}{d-1}$.

Since $\frac{a_{\mathrm{fo}}-a_{\mathrm{f}}}{(d-1) \ln q}$ differs only from $\frac{e_{\mathrm{fo}}-e_{\mathrm{f}}}{d-1}$ by a Lipschitz function of $\beta_{s}$, namely the function $\lim _{\Omega \uparrow \mathbb{L}} \frac{1}{\|\Omega\|((d-1) \ln q}\left[\ln \mathcal{Z}_{\mathrm{f}}(\Omega)-\ln \mathcal{Z}_{\mathrm{fo}}(\Omega)\right]$, it will satisfy the same properties (the intersecting point slightly changed) provided the Lipschitz constant of this last function is sufficiently small. Indeed,

$$
\frac{a_{\mathrm{fo}}-a_{\mathrm{f}}}{(d-1) \ln q}=\frac{e_{\mathrm{fo}}-e_{\mathrm{f}}}{d-1}-\lim _{\Omega \uparrow \mathbb{L}} \frac{1}{\|\Omega\|(d-1) \ln q}\left[\ln \mathcal{Z}_{\mathrm{fo}}(\Omega)-\ln \mathcal{Z}_{\mathrm{f}}(\Omega)\right]
$$

By virtue of Lemma 3.7 the needed condition on the Lipschitz constant is $2 \frac{a_{c} \kappa e^{-\alpha}}{1-a_{c} \kappa e^{-\alpha}}<1$ since the Lipschitz constant of $\frac{e_{\mathrm{o}}-e_{\mathrm{f}}}{d-1}$ is 1 . This last inequality requires $a_{c} \kappa e^{-\alpha}<1 / 3$ and is actually fulfilled under the hypotheses of the Theorem 3.6 by noticing that the geometric constant $\kappa^{\prime}=3^{d-1}-1 \geq 8$, these hypotheses imply $a_{c} \kappa e^{-\alpha} \leq a_{c} c / \kappa^{\prime} \leq a_{c} c / 8$ where the last term is less than $1 / 3$ for $c \leq 0.7$.

\section{Acknowledgments}

The authors thank S. Miracle-Solé, S. Shlosman, and V. Zagrebnov for helpful discussions. L.L. acknowledges the warm hospitality and financial support of the Centre de Physique Théorique. 


\section{Appendix: Proof of Proposition 3.3}

We begin the proof by considering contours $\Gamma=\left\{\operatorname{supp} \Gamma, X^{\Gamma}, Y^{\Gamma}\right\}$ (where $\left\{X^{\Gamma}, Y^{\Gamma}\right\}$ is the configuration having $\Gamma$ as unique contour) without polymers, i.e. $Y^{\Gamma}=\emptyset$. We let $X_{s}^{\Gamma}=X^{\Gamma} \cap B\left(\mathbb{L}_{0}\right)$ be the bonds of $X^{\Gamma}$ that belong to the boundary layer and $X_{b}^{\Gamma}=X^{\Gamma} \backslash X_{s}^{\Gamma}$. A site $i \in \mathbb{L}_{0}$ of the boundary layer will be called regular if the bonds of its envelope that belong to the boundary layer are either all empty or all occupied. It will be called irregular otherwise and we will denote by $I_{0}(\Gamma)$ the set of irregular sites of the contour $\Gamma$. Namely $I_{0}(\Gamma)=\left\{i \in \mathbb{L}_{0}: 1 \leq\left|E(i) \cap X_{s}^{\Gamma}\right| \leq 2(d-1)-1\right\}$.

Lemma A.1

$$
\left|\varrho\left(\Gamma^{p}\right)\right| q^{\underline{e}\left\|\Gamma^{p}\right\|} \leq q^{-\frac{\left|I_{0}\left(\Gamma^{p}\right)\right|}{2(d-1)}-\left(\frac{1}{d}-\beta_{b}\right)\left|X_{b}^{\Gamma^{p}}\right|}
$$

Proof. By Lemma 3.2 and definition (3.9), one has

$$
\begin{aligned}
\varrho\left(\Gamma^{p}\right) q^{\underline{e}\left\|\Gamma^{p}\right\|} & =q^{-\left(H_{\operatorname{supp} \Gamma}^{\operatorname{dil}}(X)-\underline{e}\left\|\Gamma^{p}\right\|\right)} \\
& =q^{\sum_{i \in \operatorname{supp} \Gamma^{p} \cap B\left(\mathbb{L}_{0}\right)} \frac{\beta_{s}}{2}\left|E(i) \cap X_{s}\right|+\beta_{b}\left|X_{b}\right|-S(X)+C(X)-\chi_{p}+\underline{e}\left\|\Gamma^{p}\right\|}
\end{aligned}
$$

Here, $\chi_{\mathrm{f}}=0, \chi_{\mathrm{o}}=1$, and to simplify formulae we put hereafter $X, X_{s}$, and $X_{b}$ instead of $X^{\Gamma^{p}}, X_{s}^{\Gamma^{p}}$, and $X_{b}^{\Gamma^{p}}$. By using the relations

$$
\begin{aligned}
& S(X)=S\left(X_{s}\right)+S\left(X_{b} \cup X_{s}\right)-S\left(X_{s}\right) \\
& C(X)=C\left(X_{s}\right)+C\left(X_{b} \cup X_{s}\right)-C\left(X_{s}\right)
\end{aligned}
$$

we get

$$
\varrho\left(\Gamma^{p}\right) q^{\underline{e}\left\|\Gamma^{p}\right\|} \leq q^{\mathbf{A}_{s}\left(\Gamma^{p}\right)+\mathbf{A}_{b}\left(\Gamma^{p}\right)}
$$

where

$$
\begin{aligned}
& \mathbf{A}_{s}\left(\Gamma^{p}\right)=\sum_{i \in \operatorname{supp} \Gamma^{p} \cap B\left(\mathbb{L}_{0}\right)}\left(\frac{\beta_{s}}{2}\left|E(i) \cap X_{s}\right|-|i \cap S(X)|+\underline{e}\right)+C\left(X_{s}\right) \\
& \mathbf{A}_{b}\left(\Gamma^{p}\right)=\beta_{b}\left|X_{b}\right|-S\left(X_{b} \cup X_{s}\right)+S\left(X_{s}\right)+C\left(X_{b} \cup X_{s}\right)-C\left(X_{s}\right)
\end{aligned}
$$

Let us first bound $\mathbf{A}_{s}\left(\Gamma^{p}\right)$.

Consider an irregular site (of the boundary layer). Then by definition: $1 \leq\left|E(i) \cap X_{s}\right| \leq 2(d-1)-1$. Since $\underline{e}=\min \left\{e_{\mathrm{f}}=0, e_{\mathrm{fo}}=1-(d-1) \beta_{s}\right\}$, 
we have,

$$
\begin{aligned}
\frac{\beta_{s}}{2}\left|E(i) \cap X_{s}\right|-|i \cap S(X)|+\underline{e} & =\frac{\beta_{s}}{2}\left|E(i) \cap X_{s}\right|-1+\underline{e} \\
& \leq \frac{1-\underline{e}}{2(d-1)}\left|E(i) \cap X_{s}\right|-1+\underline{e} \\
& =(1-\underline{e}) \frac{\left|E(i) \cap X_{s}\right|-2(d-1)}{2(d-1)} \\
& \leq \frac{\left|E(i) \cap X_{s}\right|-2(d-1)}{2(d-1)}
\end{aligned}
$$

where we used successively $\beta_{s} \leq(1-e) /(d-1)$ and $\underline{e} \leq 0$

Next, we take into account that the number of connected components may be bounded as (c.f. e.g. [19, 22]):

$$
C(X) \leq \sum_{i: 1 \leq\left|E(i) \cap X_{s}\right| \leq d-1} \frac{1}{2^{\left|E(i) \cap X_{s}\right|}}
$$

When $1 \leq\left|E(i) \cap X_{s}\right| \leq 2(d-1)-1$, we infer

$$
\frac{\left|E(i) \cap X_{s}\right|-2(d-1)}{2(d-1)}+\frac{\chi\left(1 \leq\left|E(i) \cap X_{s}\right| \leq d-1\right)}{2^{\left|E(i) \cap X_{s}\right|}} \leq \frac{-1}{2(d-1)}
$$

so that each irregular site $i$ of the boundary layer provides at most a contribution $\frac{-1}{2(d-1)}$ to the right-hand side of (A.6) giving

$$
q^{\mathbf{A}_{s}\left(\Gamma^{p}\right)} \leq q^{-\frac{\left|I_{0}\left(\Gamma^{p}\right)\right|}{2(d-1)}}
$$

Consider now, the quantity $\mathbf{A}_{b}\left(\Gamma^{p}\right)$. One has

$$
q^{\mathbf{A}_{b}\left(\Gamma^{p}\right)} \leq \prod_{\delta} q^{\beta_{b}|\delta|-S\left(\delta \cup X_{s}\right)+S\left(X_{s}\right)+C\left(\delta \cup X_{s}\right)-C\left(X_{s}\right)}
$$

where the product runs over connected component of $X_{b}$. By the same geometrical observation as in the proof of Theorem 2.1. one easily gets the inequality

$$
\frac{|\delta|}{d}-S\left(\delta \cup X_{s}\right)+S\left(X_{s}\right)+C\left(\delta \cup X_{s}\right)-C\left(X_{s}\right) \leq 0
$$


that gives

$$
q^{\mathbf{A}_{b}\left(\Gamma^{p}\right)} \leq q^{-\left(\frac{1}{d}-\beta_{b}\right)\left|X_{b}\right|}
$$

which in turn implies the lemma by taking into account (A.5) and A.11).

Notice that by (A.2) one has the following bound

$$
\frac{\partial}{\partial \beta_{s}} \ln \varrho\left(\Gamma^{p}\right) \leq\left\|\Gamma^{p}\right\|(d-1) \ln q
$$

Considering still contours $\Gamma=\left\{\sup \Gamma, X^{\Gamma}, Y^{\Gamma}\right\}$ without polymers, i.e. $\left(Y^{\Gamma}=\emptyset\right)$ we have the

Lemma A.2 Assume that $\beta_{b}<\frac{1}{d}$, and $q^{\frac{1}{d}-\beta_{b}}>2 \nu_{d}=2(2 d)^{2}$, then

$$
\sum_{\Gamma^{p}: \operatorname{supp} \Gamma^{p}=S} \varrho\left(\Gamma^{p}\right) q^{\underline{e}\left\|\Gamma^{p}\right\|} \leq\left(2^{2 d-1} q^{-\frac{1}{2(d-1)}}+8 \nu_{d} q^{-\left(\frac{1}{d}-\beta_{b}\right)}\right)^{\|S\|} \frac{1}{1-2 \nu_{d} q^{-\left(\frac{1}{d}-\beta_{b}\right)}}
$$

which shows that, whenever $q$ is large enough, the Peierls condition holds true for the class of contours without polymers.

Proof. First, observe that for contours $\Gamma^{p}$ with support supp $\Gamma^{p}=S$ and number of irregular sites of the boundary layer $\left|I_{0}\left(\Gamma^{p}\right)\right|=k$ one has $\left|X_{b}\right|=\left|\delta_{1}\right|+\ldots+\left|\delta_{m}\right| \geq\|S\|-k$. Therefore,

$$
\begin{aligned}
\sum_{\Gamma^{p}: \text { supp } \Gamma^{p}=S} \varrho\left(\Gamma^{p}\right) q^{e}\left\|\Gamma^{p}\right\| \leq & \sum_{0 \leq k \leq\|S\|} \sum_{\Gamma^{p}:\left|I_{0}\left(\Gamma^{p}\right)\right|=k} q^{-\frac{k}{2(d-1)}} q^{-\left(\frac{1}{d}-\beta_{b}\right)\left|X_{b}\right|} \\
\leq & \sum_{0 \leq k \leq\|S\|}\left(\begin{array}{c}
\|S\| \\
k
\end{array}\right) 2^{2(d-1) k} 2^{\|S\|-k} q^{-\frac{k}{2(d-1)}} \\
& \times \sum_{n \leq\|S\|} \sum_{\substack{i_{1}, \ldots, i_{n} ; \\
i_{\alpha} \in S \cap \mathbb{L}_{0}\left|\delta_{1}\right|+\ldots+\left|\delta_{n}\right| \geq\|S\|-k}} \sum_{\substack{\delta_{1} ; i_{1}, \ldots, \delta_{n} \gg i_{n} \\
j=1}}^{m} \prod^{-\left(\frac{1}{d}-\beta_{b}\right)\left|\delta_{j}\right|}
\end{aligned}
$$

Here the binomial coefficient $\left(\begin{array}{c}\|S\| \\ k\end{array}\right)$ bounds the choice of irregular sites of the boundary layer while the factor $2^{2(d-1) k} 2^{\|S\|-k}$ bounds the numbers of contours with $\|S\|$ lines and $k$ irregular sites. Then 


$$
\begin{gathered}
\sum_{\Gamma^{p}: \operatorname{supp} \Gamma^{p}=S} \varrho\left(\Gamma^{p}\right) q^{\underline{e}\left\|\Gamma^{p}\right\|} \leq \sum_{0 \leq k \leq\|S\|}\left(\begin{array}{c}
\|S\| \\
k
\end{array}\right) 2^{2(d-1) k} 2^{\|S\|-k} q^{-\frac{k}{2(d-1)}} \\
\times \sum_{n \leq\|S\|}\left(\begin{array}{c}
\|S\| \\
n
\end{array}\right) \sum_{m_{1}+\ldots+m_{n} \geq\|S\|-k} \prod_{j=1}^{n}\left(\nu_{d} q^{-\left(\frac{1}{d}-\beta_{b}\right)}\right)^{m_{j}}
\end{gathered}
$$

Here the binomial coefficient $\left(\begin{array}{c}\|S\| \\ n\end{array}\right)$ bounds the choice for the components $\delta_{1}, \ldots, \delta_{n}$ of $X_{b}$ to hit the boundary layer at $i_{1}, \ldots, i_{n}$. The above inequality yields

$$
\begin{gathered}
\sum_{\Gamma^{p}: \operatorname{supp} \Gamma^{p}=S} \varrho\left(\Gamma^{p}\right) q^{\underline{e}\left\|\Gamma^{p}\right\|} \leq \sum_{0 \leq k \leq\|S\|}\left(\begin{array}{c}
\|S\| \\
k
\end{array}\right) 2^{2(d-1) k} 2^{\|S\|-k} q^{-\frac{k}{2(d-1)}} \\
\times \sum_{n \leq\|S\|}\left(\begin{array}{c}
\|S\| \\
n
\end{array}\right) \sum_{m \geq\|S\|-k}\left(2 \nu_{d} q^{-\left(\frac{1}{d}-\beta_{b}\right)}\right)^{m} \\
\leq \sum_{0 \leq k \leq\|S\|}\left(\begin{array}{c}
\|S\| \\
k
\end{array}\right) 2^{2(d-1) k} 2^{\|S\|-k} q^{-\frac{k}{2(d-1)}} \\
\times\left(2 \nu_{d} q^{-\left(\frac{1}{d}-\beta_{b}\right)}\right)^{\|S\|-k} \frac{2^{\|S\|}}{1-2 \nu_{d} q^{-\left(\frac{1}{d}-\beta_{b}\right)}}
\end{gathered}
$$

that gives the inequality of the lemma.

We now turn to the general case of contours with non empty polymers and first give a bound on the activity $\psi(\gamma)$ of polymers.

Lemma A.3 Assume that $\beta_{b}<\frac{1}{d}$, and $c \nu_{d}^{2} q^{-\frac{1}{d}+\beta_{b}} \leq 1$ with $c=8 e(e-$ 1) $(\sqrt{3}+2) e^{\frac{2}{\sqrt{3}+1}}$ and $\nu_{d}=(2 d)^{2}$, then

$$
|\psi(\gamma)| \leq\left(c \nu_{d}^{2} q^{-\frac{1}{d}+\beta_{b}}\right)^{|\gamma|}
$$

Proof. Let us first recall the definition (3.6): $\psi(\gamma) \equiv \sum_{A: \operatorname{supp} A=\gamma} \omega(A)$ where the weights of aggregates are defined by (see (3.3) and $(\underline{3.4}): \omega(A)=$ $\prod_{\gamma \in A} e^{-\Phi(\gamma)}-1$. By Theorem 2.1] we know that $|\Phi(\gamma)| \leq\left(e c_{0} \nu_{d} q^{-\frac{1}{d}+\beta_{b}}\right)^{|\gamma|}(\leq$ 1) for $q$ large enough. Since for any $|x| \leq 1,\left|e^{-x}-1\right| \leq(e-1)|x|$, we have

$$
|\Psi(\gamma)|=\left|e^{-\Phi(\gamma)}-1\right| \leq(e-1)|\Phi(\gamma)| \leq\left((e-1) e c_{0} \nu_{d} q^{-\frac{1}{d}+\beta_{b}}\right)^{|\gamma|} \equiv e^{-\sigma|\gamma|}
$$


Then,

$$
\begin{aligned}
\sum_{A: \operatorname{supp} A=\gamma}|\omega(A)| & =\sum_{n \geq 1} \sum_{\substack{\gamma_{1}, \ldots, \gamma_{n} \\
\operatorname{supp}\left\{\gamma_{1}, \ldots, \gamma_{n}\right\}=\gamma}} \prod_{j=1}^{n}\left|\Psi\left(\gamma_{j}\right)\right| \\
& \leq \sum_{n \geq 1} 2^{|\gamma|} \sum_{\substack{\gamma_{1} \beta_{1}, \ldots, \gamma_{n} b_{n} \\
\operatorname{supp}\left\{\gamma_{1}, \ldots, \gamma_{n}\right\}=\gamma}} \prod_{j=1}^{n} e^{-\sigma\left|\gamma_{j}\right|} \\
& \leq \sum_{n \geq 1} 2^{|\gamma|} \sum_{\substack{m_{1}, \ldots, m_{n} \\
m_{1}+\ldots+m_{n} \geq|\gamma|}} \prod_{j=1}^{n}\left(\nu_{d} e^{-\sigma}\right)^{m_{j}} \\
& \leq \sum_{n \geq 1} \sum_{\substack{m_{1}, \ldots, m_{n} \\
m_{1}+\ldots+m_{n} \geq|\gamma|}} \prod_{j=1}^{n}\left(2 \nu_{d} e^{-\sigma}\right)^{m_{j}}
\end{aligned}
$$

Here, we used as in the proof of Theorem 2.1 that the number of polymers of length $m$ containing a given bond or a given vertex is less than $\nu_{d}^{m}$; the term $2^{|\gamma|}$ bounds the combinatoric choice of the bonds or vertices $b_{j} \in \gamma_{j}$, because $\gamma$ being connected, it contains $n-1$ such intersecting cells (see [13]).

We put $k=m_{1}+\ldots+m_{n}$ and notice that there are at most $\left(\begin{array}{c}k \\ n-1\end{array}\right)$ such numbers to get

$$
\begin{aligned}
\sum_{A: \operatorname{supp} A=\gamma}|\omega(A)| & =\sum_{1 \leq n \leq k} \sum_{k \geq|\gamma|}\left(\begin{array}{c}
k \\
n-1
\end{array}\right)\left(2 \nu_{d} e^{-\sigma}\right)^{k} \\
& \leq \sum_{k \geq|\gamma|}\left(4 \nu_{d} e^{-\sigma}\right)^{k}=\sum_{k \geq|\gamma|}\left(\frac{c}{2} \nu_{d}^{2} q^{-\frac{1}{d}+\beta_{b}}\right)^{k} \\
& \leq \frac{1}{1-\frac{c}{2} \nu_{d}^{2} q^{-\frac{1}{d}+\beta_{b}}}\left(\frac{c}{2} \nu_{d}^{2} q^{-\frac{1}{d}+\beta_{b}}\right)^{|\gamma|}
\end{aligned}
$$

provided that $\frac{c}{2} \nu_{d}^{2} q^{-\frac{1}{d}+\beta_{b}}<1$. The lemma then follows by assuming that $\frac{c}{2} \nu_{d}^{2} q^{-\frac{1}{d}+\beta_{b}} \leq \frac{1}{2}$

We finally turn to the

\section{Proof of Proposition 3.3}

Consider a contour $\Gamma=\left\{\operatorname{supp} \Gamma, X^{\Gamma}, Y^{\Gamma}\right\}$ and as above the decomposition $X^{\Gamma}=X_{s}^{\Gamma} \cup X_{b}^{\Gamma}$ where $X_{s}^{\Gamma}=X^{\Gamma} \cap B\left(\mathbb{L}_{0}\right)$ is the set of bonds of $X^{\Gamma}$ on the boundary layer and $X_{b}^{\Gamma}=X^{\Gamma} \backslash X_{s}^{\Gamma}$. Consider also the union $Z_{b}=X_{b}^{\Gamma} \cup Y^{\Gamma}$. 
Notice that the set $Z=X_{s}^{\Gamma} \cup Z_{b}$ is a family of hydras and there are at most $3^{\left|Z_{b}\right|}$ contours corresponding to this family: this is because a bond in $Z_{b}$ may be occupied either by $X_{b}^{\Gamma}$ or by $Y^{\Gamma}$ or by both. Let

$$
|\widetilde{\varrho}(Z)|=\sum_{\Gamma: X_{b}^{\Gamma} \cup Y^{\Gamma}=Z}|\varrho(\Gamma)|
$$

The above remark on the number of contours associated to $Z$ and Lemma A.3 implies

$$
\begin{aligned}
|\varrho(\Gamma)| q^{e}\|\Gamma\| & \leq q^{-\frac{\left|I_{0}(\Gamma)\right|}{2(d-1)}}\left(3 \sup \left\{q^{-\left(\frac{1}{d}-\beta_{b}\right)}, c \nu_{d}^{2} q^{-\frac{1}{d}+\beta_{b}}\right\}\right)^{\left|Z_{b}\right|} \\
& \leq q^{-\frac{\left|I_{0}(\Gamma)\right|}{2(d-1)}}\left(3 c \nu_{d}^{2} q^{-\frac{1}{d}+\beta_{b}}\right)^{\left|Z_{b}\right|}
\end{aligned}
$$

The rest of the proof is then analog to that of Lemma A.2 starting from Lemma A.1 and replacing $\nu_{d} q^{-\frac{1}{d}+\beta_{b}}$ by $3 c \nu_{d}^{3} q^{-\frac{1}{d}+\beta_{b}}$. It gives

$\sum_{\Gamma^{p}: \operatorname{supp} \Gamma^{p}=S}\left|\varrho\left(\Gamma^{p}\right)\right| q^{\underline{\underline{e}}\left\|\Gamma^{p}\right\|} \leq\left(2^{2 d-1} q^{-\frac{1}{2(d-1)}}+24 c \nu_{d}^{3} q^{-\frac{1}{d}+\beta_{b}}\right)^{\|S\|} \frac{1}{1-6 c \nu_{d}^{3} q^{-\frac{1}{d}+\beta_{b}}}$

provided $6 c \nu_{d}^{3} q^{-\frac{1}{d}+\beta_{b}}<1$ and ends the proof of the proposition.

\section{References}

[1] D.B. Abraham, Surface structures and phase transitions - exact results in in "Phases Transitions and Critical Phenomena", Vol. 10, C. Domb and J.L. Lebowitz, eds, Academic Press, London, New York, 1986.

[2] P.S. Aleksandrov, Combinatorial Topology, vol. 3, Graylock Press, Albany, 1960.

[3] K. Binder, Critical behaviour at surfaces, in "Phases Transitions and Critical Phenomena", Vol. 8, C. Domb and J.L. Lebowitz, eds, Academic Press, London, New York, 1983.

[4] C. Borgs and J. Imbrie, A unified approach to phase diagrams in fields theory and statistical mechanics, Commun. Math. Phys. 123, 305 (1989). 
[5] A. Bakchich, A. Benyoussef, and L. Laanait, Phase diagram of the Potts model in an external magnetic field, Ann. Inst. Henri Poincaré 50, 17 (1989).

[6] J. Bricmont, K. Kuroda, and J. L. Lebowitz, First order phase transitions in lattice and continuous systems, Commun. Math. Phys. 101, 501-538 (1985).

[7] F. Cesi and F. Martinelli, On the layering transition of an SOS model interacting with a wall I. Equilibrium results, J. Stat. Phys. 82, 823 (1997).

[8] R. L. Dobrushin, Estimates of semi-invariants for the Ising model at low temperatures, Amer. Math. Soc. Transl. 177, 59 (1996).

[9] E.I. Dinaburg, and A.E. Mazel, Layering transition in SOS model with external magnetic field, J. Stat. Phys. 74, 533 (1994).

[10] E.I. Dinaburg, and Ya.G. Sinai, Tr. Conf. Mat. Fiz. (1984), and Selecta. Math. Sov. 7, 3 (1987).

[11] R.L. Dobrushin, R. Kotecky, and S. Shlosman, Wulff construction: a global shape from local interactions, Providence, 1992.

[12] K. Druhl and H. Wagner, Algebraic formulation of duality transformation for abelian lattice model, Ann. Phys. 141, 225 (1982).

[13] G. Gallavotti, A. Martin Löf, and S. Miracle-Solé, Some problems connected with the coexistence of phases in the Ising model, in "Statistical mechanics and mathematical problems", Lecture Notes in Physics vol 20, pp. 162, Springer, Berlin (1973).

[14] C.M. Fortuin, P.W. Kasteleyn, On the random-cluster model I: Introduction and relation to other models, Physica 57, 536 (1972).

[15] J. Fröhlich and C.E. Pfister, Semi-infinite Ising model I: Thermodynamic functions and phase diagram in absence of magnetic field, Commun. Math. Phys. 109, 493 (1987).

[16] J. Fröhlich and C.E. Pfister, Semi-infinite Ising model II: The wetting and layering transition, Commun. Math. Phys. 112, 51 (1987). 
[17] J. Fröhlich and C.E. Pfister, The wetting and layering transitions in the half-infinite Ising model, Europhys. Lett. 3, 845 (1987).

[18] P. Holicky, R. Kotecky, and M. Zahradnik, Rigid interfaces for lattice models at low temperatures, J. Stat. Phys. 50, 755 (1988).

[19] R. Kotecky, L. Laanait, A. Messager, and J. Ruiz, The q-state Potts model in the standard Pirogov-Sinai theory: surface tension and Wilson loops, J. Stat. Phys., 58, 199 (1990).

[20] R. Kotecký and D. Preiss, Cluster Expansion for Abstract Polymer Models, Commun. Math. Phys. 103491 (1986).

[21] R. Kotecký and D. Preiss, An inductive approach to Pirogov-Sinai theory, Supp. Rend. Circ. Matem. Palermo II (3), 161 (1984).

[22] L. Laanait, N. Masaif, J. Ruiz, Phase coexistence in partially symmetric q-state models, J. Stat. Phys. 72, 721 (1993).

[23] L. Laanait, A. Messager, and J. Ruiz, Phase coexistence and surface tensions for the Potts model, Commun. Math. Phys. 105, 527 (1986).

[24] L. Laanait, A. Messager, S. Miracle-Sole, J. Ruiz, and S. Shlosman, Interfaces the in Potts model I: Pirogov-Sinai theory of the FortuinKasteleyn representation, Commun. Math. Phys. 140, 81 (1991).

[25] S. Lefschetz, Introduction to Topology, Princeton University Press, Princeton, 1949.

[26] R. Lipowsky, The Semi-infinite Potts model: aA new low temperature phase, Z. Phys. B-Condenced Matter 45, 229 (1982).

[27] S. Miracle-Solé, On the convergence of cluster expansion, Physica A 279, 244 (2000).

[28] B.M. Mc Coy and T.T. Wu, The two-dimensional Ising model, Harvard University Press, Cambridge Massachusets, 1973.

[29] C.E. Pfister and O. Penrose, Analyticity properties of the surface free energy of the Ising model, Commun. Math. Phys. 115, 691 (1988). 
[30] O. Ore, The Four-Color Problem, Academic Press, New-York, London, 1967.

[31] D. Ruelle, Statistical Mechanics: Rigorous Results, Benjamin, New York Amsterdam (1969).

[32] Ya.G. Sinai, Theory of Phase Transitions: Rigorous Results, Pergamon Press, London, 1982.

[33] A.D. Sokal, Bounds on the complex zeros of (Di)chromatics polynomials and Potts-model partitions functions, Combinatorics, Probability and Computing 10, 41 (2001).

[34] M. Zahradnik, An alternate version of Pirogov-Sinai theory, Commun. Math. Phys. 93, 359 (1984).

[35] M. Zahradnik, Analyticity of low-temperature phase diagram of lattice spin models, J. Stat. Phys. 47, 725 (1987). 


\section{Figure captions}

1. The diagram of ground states.

2. A hydra, in two dimensions (a dimension not considered in this paper), with 2 feet (components of full lines), 5 necks (dotted lines), and 3 heads (components of dashed lines). 


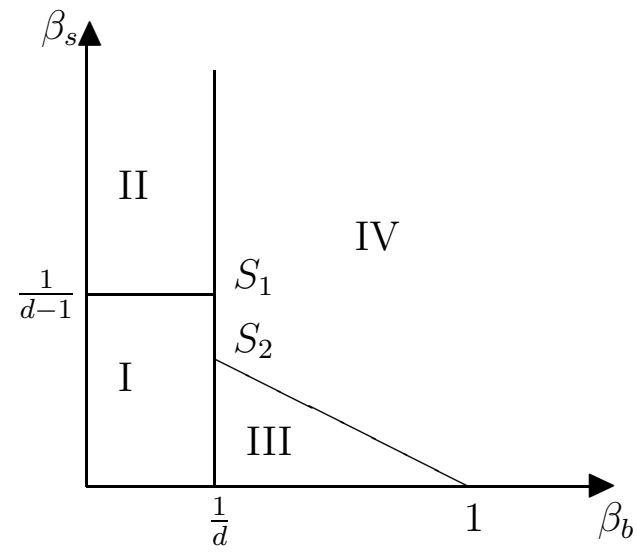




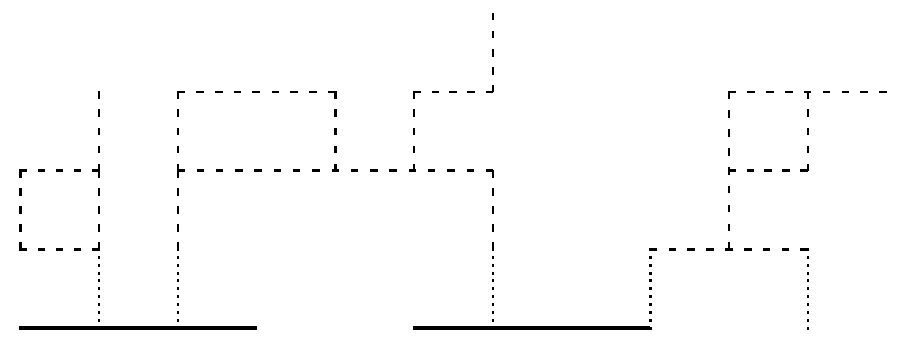

